\title{
CFD simulation of channels for direct and reverse electrodialysis
}

\author{
A. Tamburini ${ }^{\mathrm{a}}$, G. La Barbera ${ }^{\mathrm{a}}$, A. Cipollina ${ }^{\mathrm{a}}$, M. Ciofalo ${ }^{\mathrm{b}}$, G. Micale ${ }^{\mathrm{a}, *}$ \\ ${ }^{a}$ Dipartimento di Ingegneria Chimica, Gestionale, Informatica, Meccanica, Università di Palermo, Viale delle \\ Scienze Ed. 6, 90128 Palermo, Italy \\ Email: giorgiod.maria.micale@unipa.it \\ ${ }^{b}$ Dipartimento dell'Energia, Università di Palermo, Viale delle Scienze Ed. 6, 90128 Palermo, Italy
}

Received 8 May 2012; Accepted 15 June 2012

\section{A B S TRACT}

Flows within very thin channels, typically filled with spacers, can be often encountered in many processes such as electrodialysis (ED) and reverse electrodialysis (RED). Although the ED and the RED processes have been studied for a long time, the optimization of the fluid dynamics within the channels is still an open problem. In the present work, realized within the EU-FP7 funded REAPower project, computational fluid dynamics simulations were carried out in order to predict the fluid flow field inside a single ED/RED channel. Some different configurations were tested which includes: an empty channel, a channel provided with a spacer, and a channel filled with a purposely manufactured fiber porous medium. Two types of spacers were investigated: (1) a commercial type made of woven perpendicular filaments and (2) an overlapped perpendicular filament spacer. A sensitivity analysis concerning computational grid size and topology was performed. For the cases investigated, adopting the hybrid grids mainly composed of hexahedral volumes was found to be more reliable and less computational demanding than tetrahedral grids. As concerns the dependence of the pressure drops on the flow rate, the empty channel was found to guarantee the lowest pressure drops at a given fluid flow rate, as expected. Conversely, the woven spacer filled channel was found to provide larger pumping costs. The pressure drops along the channel filled with a porous medium even at low flow rate were very high thus suggesting that this specific configuration may be unsuitable and that further investigations should be devoted to this topic.

Keywords: CFD; Salinity gradient power; Renewable energy; Electrodialysis; Reverse electrodialysis; Water; Spacer

\section{Introduction}

The need of freshwater and energy from renewable resources has become an issue of crucial importance nowadays.

Electrodialysis (ED) is a desalination technique based on the transport of salt ions from a solution to another through ion-exchange membranes under the *Corresponding author. influence of an applied electrical potential difference. The outputs of an ED process are: (1) fresh water and (2) a salt-concentrated solution.

Reverse electrodialysis (RED) is the opposite process which converts the chemical potential difference between a salt-concentrated solution and a dilute solution into electric current (Salinity Gradient Power, SGP) [1]. Also in this case, ion-exchange membranes 
separate the channels in which concentrated and diluted solutions are alternatively fed. RED is one of the technologies able to retrieve energy from the mixing of two solutions [2]: e.g. pressure retarded osmosis [3], hydrocratic generators [4], and vapor pressure difference utilization [5]. RED processes have been operated up to now by adopting river water as dilute solution and seawater as concentrated solution. In accordance with Veerman et al. [6], the controlled mixing of $1 \mathrm{~m}^{3}$ of river water with a large amount of seawater within a RED device can produce $2.5 \mathrm{MJ}$ of energy and also produce $1.7 \mathrm{MJ}$ of energy when it is mixed with $1 \mathrm{~m}^{3}$ of seawater. This suggests that the SGP has a great potential: the global electricity demand is about $2 \mathrm{TW}$ and the global salinity power is estimated to be 2.6 TW [7]. The large electric resistance of the dilute compartment, which dramatically reduces the obtainable power density, has limited the development and spreading of RED technologies. Recently, Brauns [8] and Brauns et al. [9] proposed to use seawater as dilute solution and brine as concentrated solution thus (1) enhancing the driving force to the process and (2) significantly reducing the electrical resistance of the dilute compartment. According to these considerations, each desalination plant could be coupled with a RED process in order to provide both fresh water and energy.

The optimization of RED and ED stacks is based on many aspects requiring different kinds of studies: materials employed do not have to suffer from corrosion problems and, at the same time, should be able to resist to mechanical stresses; membranes should be very selective and thin in order to minimize the relevant electrical resistances; electrodic reactions should have small voltage losses; redox electrodic couples should be safe and stable; and the fluid flow inside the stack should be the most economically convenient. Concerning this last aspect, Computational Fluid Dynamics (CFD) can be used in order to investigate the flow of the solutions within the stack aiming at improving the performance of the whole process.

In particular, the selection of the type of the channel is a crucial point for the stack performance: for instance, it is necessary to find the best geometrical configuration constituting a compromise between the reduction of pressure drops along the channel and the minimization of any concentration polarization phenomenon. The EU-FP7 funded REAPower project fully addressed this issue for the case of a RED unit by aiming at the development of a multiscale approach, which just starts from the CFD analysis of spacer filled channel and fluid flow behavior.

A very few efforts have been devoted so far to the CFD investigation of channels for ED and RED pro- cesses: only Dirkse et al. [10] addressed this topic by developing a model based on potential flow theory in order to predict the 2D velocity and pressure field in flat-sheet geometries to be employed for RED applications. Channels typically employed for these processes are thinner than the ones used for other membrane separation processes as, for example, the Reverse Osmosis (RO) and the Membrane Distillation (MD) processes. Also, typical flow rates in ED and RED processes are lower than those employed in RO and MD applications. These differences suggest that the fluid flow within ED and RED channels is expected to be different and deserves proper attention.

Therefore, this work represents the first attempt to fully model by CFD the flow field inside different channels for ED and RED in order to find the relation between the channel geometrical configuration and the pressure drop.

Similar literature works concerning the fluid flow analysis within channels are relevant to other membrane separation processes, such as: $\mathrm{RO}$, ultrafiltration, and $\mathrm{MD}$, where generally a spacer is included inside the channels. As an example, Da Costa et al. [11] elaborated a semiempirical model in order to calculate pressure drops inside an overlapped spacer-filled channel. The model included viscous drag on the channel walls and spacer, form drag of the spacer and kinetic losses due to directional flow change. According to these authors, a large proportion of the fluid changes direction at each spacer mesh by following a zigzag path which is responsible for most of the pressure losses. By employing CFD simulations, Karode and Kumar [12] disproved Da Costa and co-authors claiming that the overall flow path does not follow a zigzag pattern since the bulk of the fluid does not change direction at each spacer mesh. They stated that most of the pressure drops are due to the direction change of the velocity vector across a thin transition region corresponding to the plane of spacer filaments intersection.

Therefore, studying the fluid flow behavior in other types of spacer-filled channels seems strongly advisable. As an example, no scientific efforts have been devoted so far to the CFD assessment of the flow field within woven spacer-filled channels.

The CFD modeling of spacer-filled channels hides a considerable numerical complexity mainly concerning the discretization of the domain to investigate. Different grid topologies may lead to different results $[13,14]$; the discrepancies may be crucial at the ED and RED scale.

The flow pattern inside spacer-filled channels is constituted by small and complex structures requiring a very high discretization degree, so that it is practi- 
cally difficult to simulate the whole channel because of the excessive computational requirements. In order to solve this problem, the Unit Cell approach is often used in CFD modeling of spacer-filled channel. A typical spacer-filled channel domain is formed by a large number of identical periodic cells which can be considered as repetitive units: the fluid flow inside a periodic cell is representative of the fluid flow in the channel zones sufficiently far from inlets, outlets, and lateral walls. As a consequence, simulating the fluid dynamics of a unit cell with translational periodic boundaries is a common shortcut to get information on the fluid flow in the whole channel. Notably, the flow and transport phenomena in spacer-filled channels become "periodic fully-developed" after a small number of unit cells [15] thus confirming the soundness of this simplifying approach. Different unit cells can be detected for the same spacer-filled channel domain. Li and Tung [16] addressed this issue and analyzed four different types of periodical boundary conditions that could be utilized to define a unit cell. They concluded that the type that gives the best agreement with experimental results is the one that is built from the center of a mesh in the spacer net.

The electrical nature of the ED/RED processes and the small scale of the channels suggest that alternative possibilities to the common net spacers may be well devised.

Nonwoven fiber porous spacers: These in principle can be considered suitable, provided that they are characterized by large porosity (especially when production techniques as the electro-spinning are employed; [17]) and mixing promotion capability. In fact, a medium of fibers provides a very large number of locations for the membranes and thus assuming some of the "corrugated" aspect. This is likely to promote turbulence near their surfaces and consequently, reduce concentration polarization phenomena. Also, when the "pores" in a nonwoven structure are very small (tens up to a few microns), the possibility of supporting very thin membranes appears to be feasible (the membrane can be considered thick with respect to the pore size) thus resulting into a lower membrane resistance [17]. Nonwoven fiber porous spacers can be designed in different ways. As an example, spacers made of a common net supporting two layers of nonwoven fibers may be designed: the layer bound with the anionic membrane should be made of an anionic conductive material and the layer bound with the cationic membrane should be made of a cationic conductive material. The adoption of such a similar conductive spacer was found to enhance the process performance by reducing concentration polarization, channel elec- trical resistance and shadow effect, scaling, and fouling [18-20].

Profiled or corrugated membranes: Brauns [17] and Strathmann [21] proposed the adoption of membranes with a special surface geometry, which does not require the use of spacers. Membranes, purposely corrugated or profiled, placed one next to another, form very thin channels thereby resulting in a significant increase of the effective surface area.

\section{Systems under investigation}

CFD was employed to predict the flow field within an ED/RED channel $400 \mu \mathrm{m}$ thick (a common size in small electrodialysis plants). The length and the width were equal to $10 \mathrm{~cm}$. According to the considerations provided in the former section, different configurations of this channel were investigated:

(i) empty (i.e. spacer-less) channel;

(ii) spacer-filled channel;

(iii) fiber porous medium filled channel.

(i) The empty channel should be considered as a reference case: it was studied to compare results and features relevant to the other two configurations.

(ii) Two different types of spacers were investigated: a commercial spacer (FUMATECH) (Fig. 1) made of woven filaments and a corresponding ideal spacer made of overlapped filaments (their computational reproductions can be observed in Fig. 2). This second spacer type was also investigated as similar spacers are very common and effective in other membrane applications [12,22]. Moreover, with reference to the electrical nature of ED and RED processes, this spacer may be conveniently made of an anionic and a cationic conductive layer. Notably, since fluid dynamics in RED and ED apparatuses is not well established and optimized, it is highly recommended to investigate also geometrical configurations which are not commercially available yet.

Both spacers have a distance between the two parallel filaments of $1.1 \mathrm{~mm}$, the filament diameter is of $0.2 \mathrm{~mm}$, and the angle formed between two crossing filaments is of $90^{\circ}$.

(iii) A first attempt to investigate the possibility of adopting a porous spacer in ED/RED processes was made in the present work. A suitable textile PET porous spacer (with a nonwoven fiber structure) was purposely manufactured by New Textile Technologies (NTT). Experimental investigations carried out by the manufacturer resulted in the following properties: volume porosity $\varepsilon=0.72$ and permeability $k_{\text {Darcy }}=$ $1.54 \cdot 10^{-10} \mathrm{~m}^{2}$. 


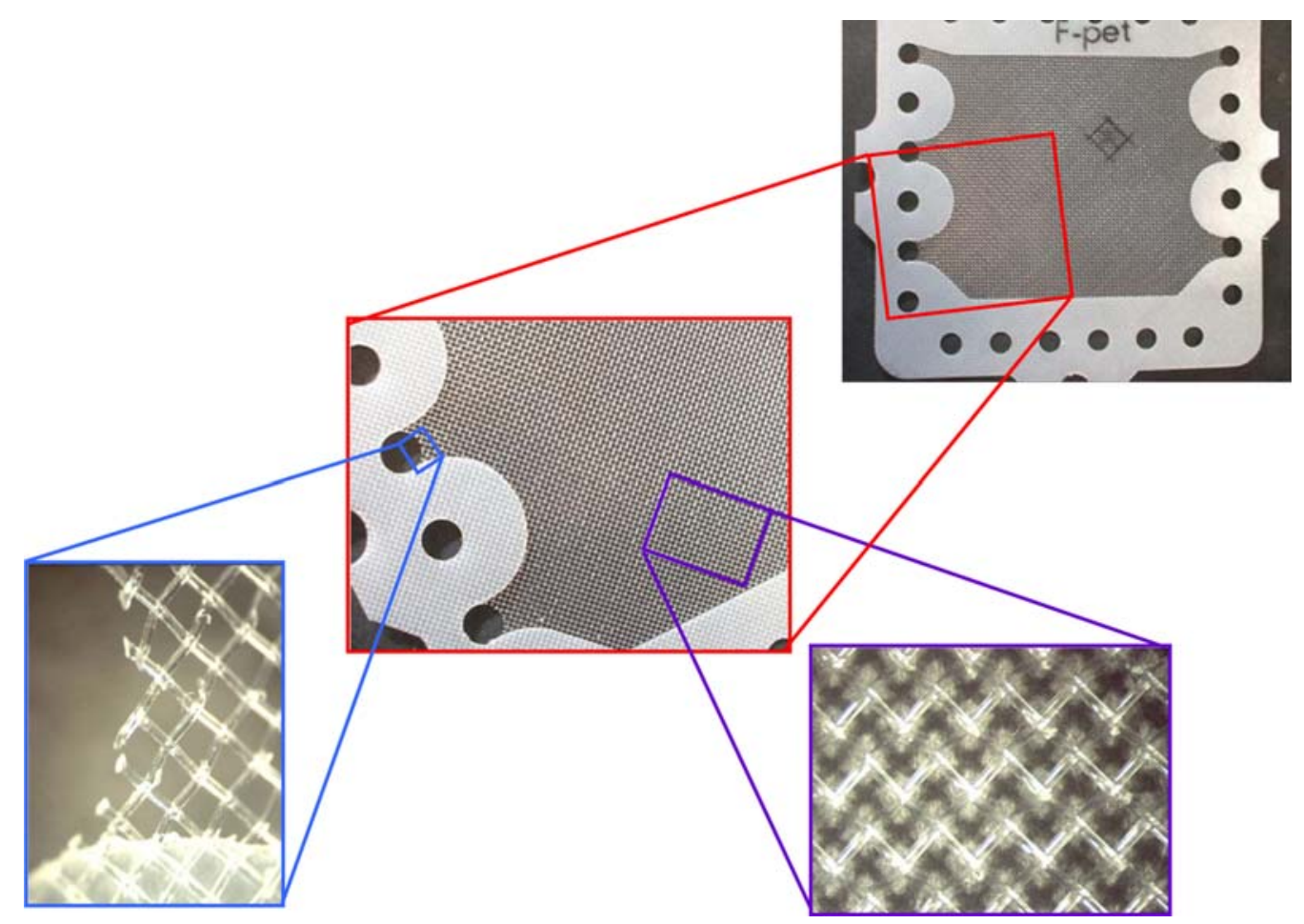

Fig. 1. FUMATECH woven spacer.
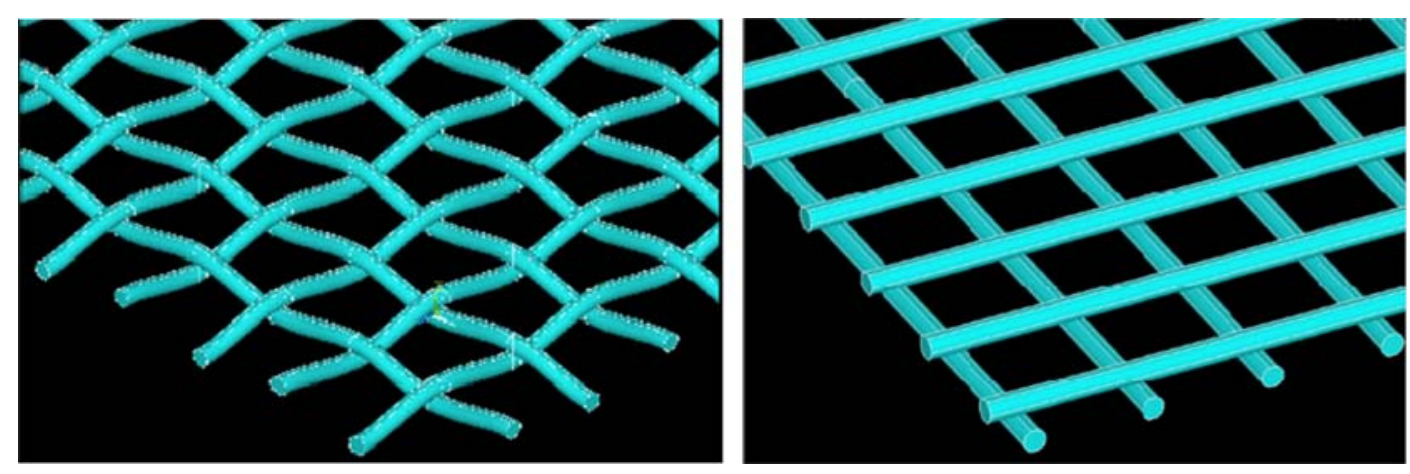

Fig. 2. Computational reproduction of the modeling of the (left) woven net spacer and the (right) overlapped one.

A number of pressure drops (see Unit Cell approach, section 4.1) corresponding to a number of average velocity values within the channel were investigated. Most attention was focused on pressure drop range typically encountered in ED and RED processes (up to about $0.1 \mathrm{bar} / \mathrm{m}$ ). However, higher pressure drops were also investigated aiming at approximately recognizing the cut-off Reynolds number at which turbulent conditions were achieved. Full details on the investigated cases can be found in section 4.1 (Table 1).

\section{Modeling}

The governing equations for three-dimensional flow of a Newtonian and incompressible fluid are the continuity and the momentum equations given by:

$$
\vec{\nabla} \cdot \vec{u}=0
$$

$\rho \frac{\partial \vec{u}}{\partial t}+\rho \vec{u} \vec{\nabla} \cdot \vec{u}=-\overrightarrow{\nabla P}+\mu \nabla^{2} \vec{u}+\vec{F}$ 


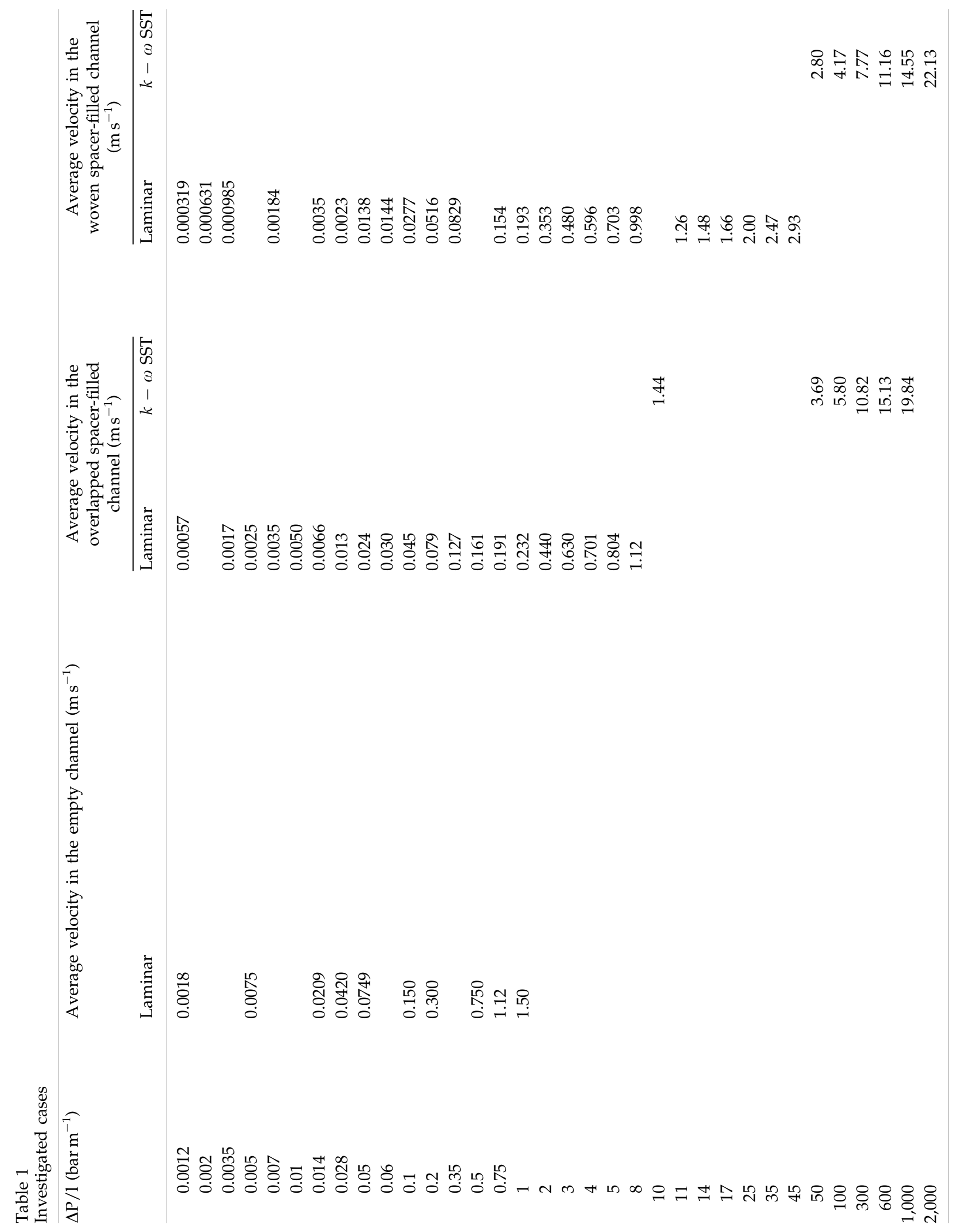


where $\mu$ is the viscosity, $\rho$ is the density, $P$ is the pressure, and $F$ is a force for volume unit which may be included in the momentum equation when the unit cell approach is employed (see section 5.4).

Typically, ED and RED apparatuses are operated at low flow rates allegedly corresponding to a laminar flow regime. These flow rates were directly simulated without employing any turbulence model. The relevant solutions were steady, thus confirming the former assumption. Only some high flow rates were simulated by including the adoption of a suitable turbulence model in order to characterize the systems under investigation also at those large Reynolds numbers, see section 6 . In this regard, Reynolds average Navier-Stokes simulations along with the $k-\omega$ SST [23] turbulence model were carried out.

Equations relevant to the $k-\omega$ SST turbulence model are not included in the present paper for brevity, full details can be found in the literature [23].

One of the channel configurations under study concerns the adoption of a fiber structure porous spacer. In this case, the pressure drops prediction was obtained by adopting Darcy's law:

$\overrightarrow{\nabla P}=-\frac{\mu}{k_{\text {Darcy }}} \vec{u}$

where $k_{\text {Darcy }}$ is the permeability or Darcy's constant.

In order to better characterize the three ED/RED channel configurations under study, the dependence of the friction coefficient on Reynolds number was also investigated. The friction coefficient $f$ was defined as:

$f=\frac{(\Delta P / l) d_{\mathrm{h}}}{\rho \vec{u}^{2} / 2}$

where $\bar{u}$ is the average velocity in the unit cell (see section 4.1) and $d_{\mathrm{h}}$ is the hydraulic diameter of the channel defined as:

$d_{\mathrm{h}}=\frac{4 \cdot \text { computational volume }}{\text { wet surface area }}$

The Reynolds number is defined as:

$\operatorname{Re}=\frac{\rho \bar{u} d_{\mathrm{h}}}{\mu}$

\section{Numerical details}

The model equations were solved by using the CFD code Ansys-CFX 13. The high resolution scheme was used to discretize the advection terms, while a coupled algorithm is used for pressure-velocity coupling. The fluid used was water at $25^{\circ} \mathrm{C}$, with a constant density $\left(997 \mathrm{~kg} / \mathrm{m}^{3}\right)$ and viscosity $\left(0.0008899 \mathrm{~kg} \mathrm{~m}^{-1} \mathrm{~s}^{-1}\right)$.

\subsection{Unit cell approach}

The geometry of the two net spacers was computationally reproduced (Fig. 2), but the discretization of the whole channel filled with these nets would require a large number of computational grid volumes thus resulting into excessive computational requirements and times.

As a consequence, the Unit Cell approach was adopted. This kind of modeling is widely used in CFD studies [15] and completely accepted by scientific community. As it can be seen in Fig. 3, different unit cells could be devised for the same spacer net. This issue was already investigated by Li and Tung [16] who found the best agreement with experimental data for the case of the unit cell type-I. According to their findings, the same unit cell type was adopted in the present work.

Translational periodic boundary conditions were imposed to velocities on the surfaces perpendicular to the fluid flow direction (Z-axis) and also on the lateral surfaces perpendicular to the $X$-axis. The filaments surfaces and the surfaces perpendicular to the $Y$-axis (membrane surfaces) were defined as walls and noslip boundary conditions were imposed on them.

The same unit cell was adopted for all the channel configurations for comparison purposes. Its size was devised from the case of the two spacer-filled channels: it is a parallelepiped with a square base. The square edge was $1.556 \mathrm{~mm}$ long, while the parallelepiped height was clearly as that of the channel, i.e. $400 \mu \mathrm{m}$.

The unit cells for the analyzed channels are shown in Fig. 4.

As far as the discretization of the computational volume is concerned, each type of channel was discretized by two different grid topologies: one tetrahedral and one hexahedral. Actually, the net spacer-filled channels grids were hybrid because of their intrinsic geometrical complexity: the whole computational domain was divided into hexahedral volumes with the exception of small zones surrounding the filaments which were discretized by tetrahedral volumes. More precisely, a parallelepiped was built around every filament and this space was discretized by tetrahedral volumes. Notably, generating these grids mainly composed by hexahedral volumes is an operation that requires a preliminary division of the 


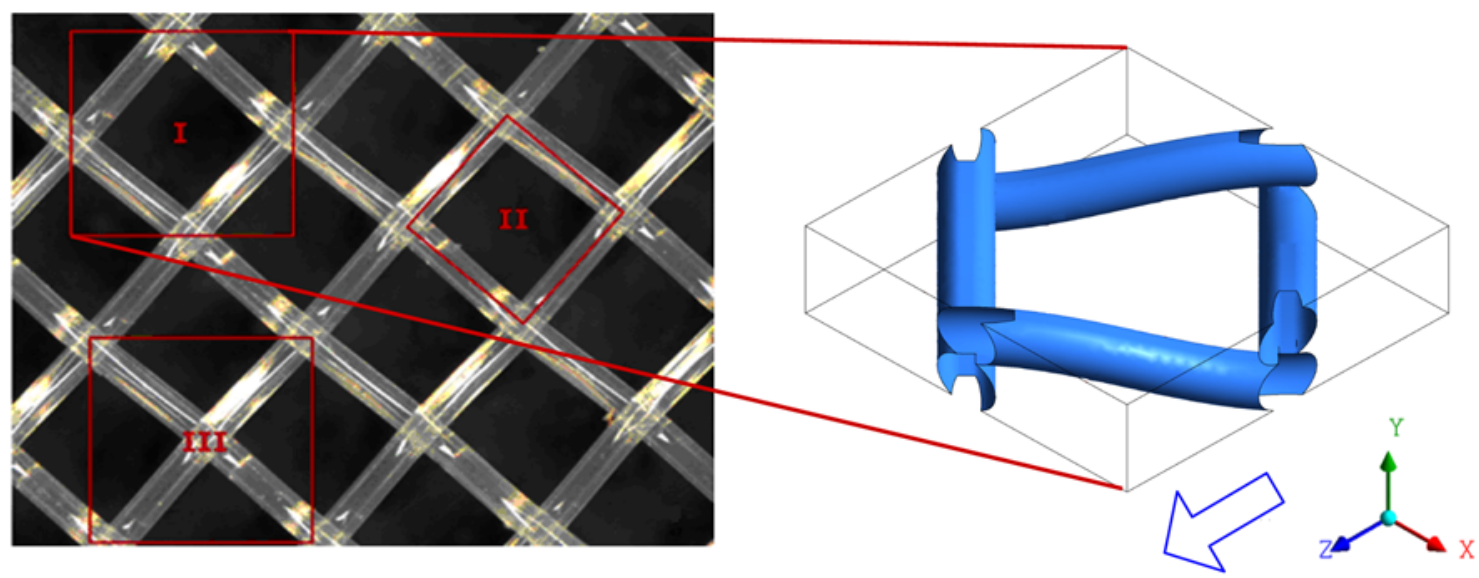

Fluid flow direction

Fig. 3. Different types of unit cell for the woven spacer.
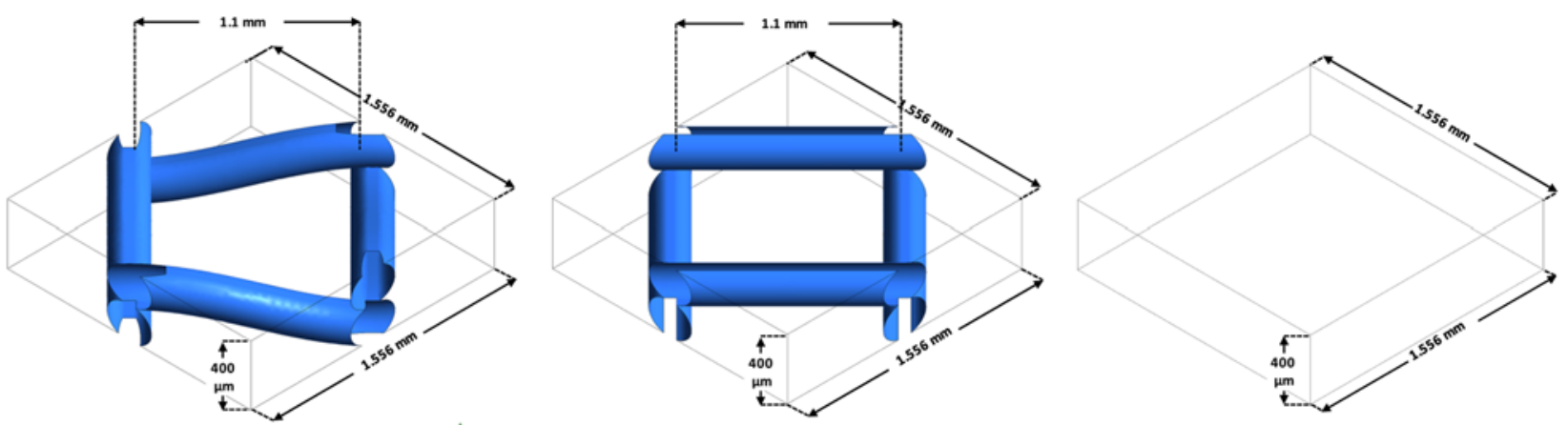

Fig. 4. Unit cell for the (a) woven spacer-filled channel, (b) overlapped spacer-filled channel, and (c) empty/porous medium filled channel.

computational domain in many subdomains (30 for the case of the overlapped spacer and 26 for the case of the woven one). The percentage of tetrahedral volumes, defined as the ratio between the volume discretized with tetrahedrons and the total volume, is $6.2 \%$ for the overlapped spacer-filled channel and $24.3 \%$ for the woven spacer-filled channel. The amount of tetrahedral volumes is bigger for the woven structure because filaments have a sinusoidal trend and the parallelepiped around the filaments is larger than the one built for the overlapped spacer filaments. The computational grids (both the tetrahedral and the hybrid one) employed for the simulation of the unit cell of two spacers-filled channels are shown in Figs. 5 and 6. Notably, an analysis of the results sensitivity on grid size was preliminary performed as will be discussed in section 5.1.

The adoption of the unit cell approach requires that a pressure gradient is imposed along the $Z$-axis in order to allow the liquid to move inside the cell (relevant full details can be found in section 5.4).
Table 1 shows all the pressure gradients imposed in the unit aell approach in order to investigate a corresponding wide range of velocities.

\section{Sensitivity analysis}

The most significant numerical aspects of the simulations, addressed in this section by a sensitivity analysis, are:

- the computational mesh size;

- the grid topology;

- the simulation type, i.e. transient or steady state; and

- the way of imposing the driving pressure gradient.

\subsection{Grid size sensitivity}

The grid sensitivity analysis of the results was addressed by comparing several computational grids 

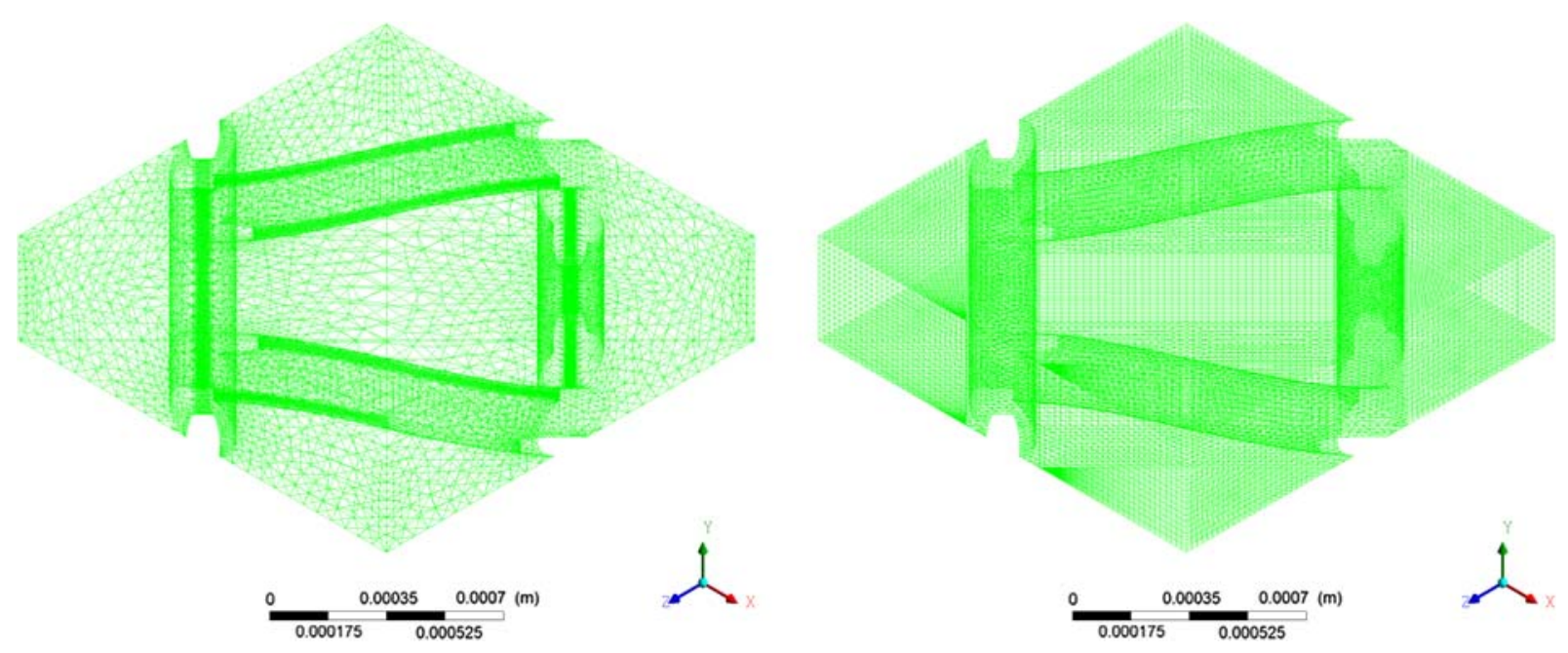

Fig. 5. Woven spacer-filled channel unit cell discretization ( $250^{\prime} 000$ volumes) by (Left) tetrahedral volumes and (Right) hybrid volumes.
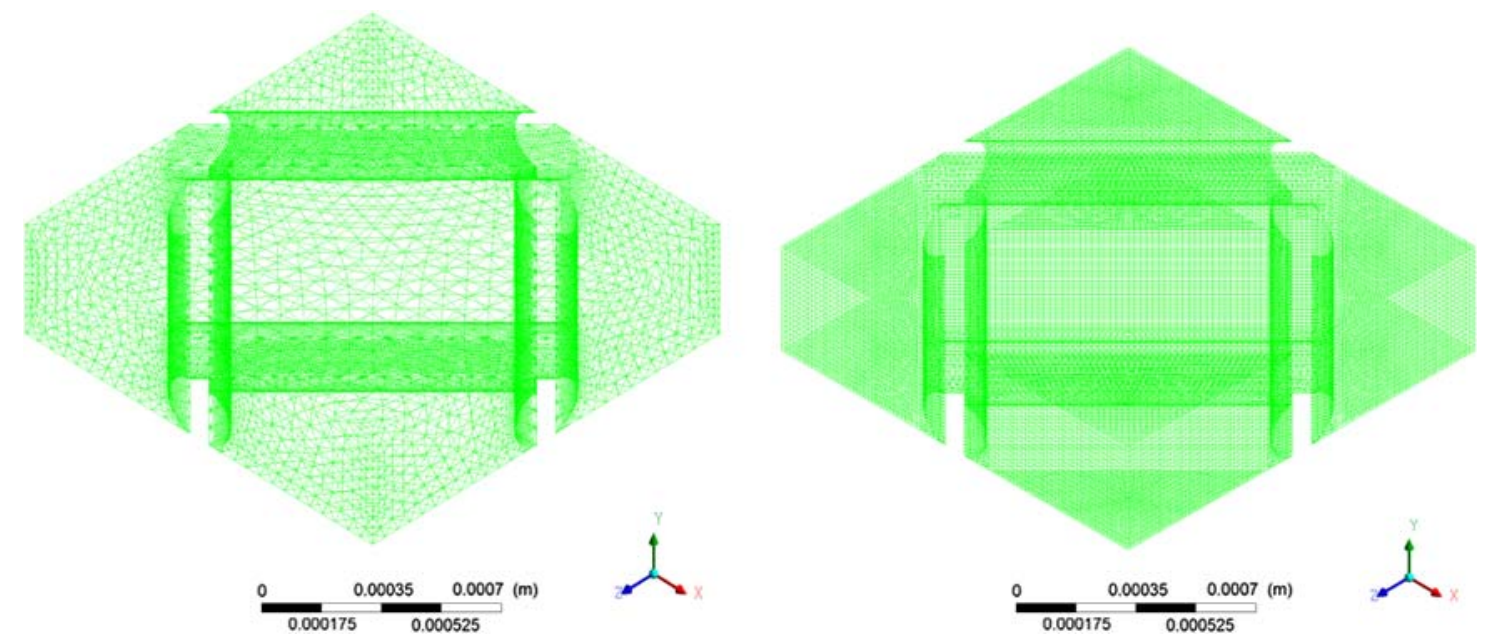

Fig. 6. Overlapped spacer-filled channel unit cell discretization ( 250 '000 volumes) by (Left) tetrahedral volumes and (Right) hybrid volumes.

reported in Table 2 for all channel-spacer configurations and for tetrahedral and hexahedral/hybrid grids (see next section).

The grid size sensitivity for the case of the empty/ porous channel will be discussed in the next section. For the spacer-filled channels, both local and global quantities are compared in the following.

As concerns local quantities, the results on three different monitoring lines (see Fig. 7) parallel to the main flow direction were analyzed. However, only the results relevant to the "medium" and the "high" lines are shown, as the "low" line results are the translated versions of those relevant to the high line.

As an example, Figs. 8 and 9 show velocity and pressure profiles along the "medium" and "high" lines for the case of the woven-spacer unit cell discretized with tetrahedral volumes.

Velocity and pressure profiles are quite similar with the exception of the coarsest grids A, B, and C (i.e. 9,735, 22,928, and 47,125 volumes). A close inspection of the velocity and pressure profiles suggests that a computational grid of $\sim 100,000$ (grid D) volumes is sufficiently fine for the grid effect on the results to be considered negligible. However, the analysis of the average velocity in the whole domain as a function of the number of computational volumes, Fig. 10(a), shows that discrepancies up to $10 \%$ with respect to the finest grids are obtained. Similar results were obtained for the overlapped configuration, see Fig. 10 (b). Finally, as partially shown in Fig. 10 and better 
Table 2

Summary of the computational grids employed for the grid independence analysis

\begin{tabular}{|c|c|c|c|c|c|c|c|c|c|c|c|c|}
\hline \multirow[t]{2}{*}{ Channel type } & \multicolumn{12}{|c|}{ Number of computational volumes } \\
\hline & \multicolumn{7}{|c|}{ Tetrahedral grids } & \multicolumn{5}{|c|}{ Hexahedral or hybrid grids } \\
\hline Grid Id & A & $\mathrm{B}$ & $\mathrm{C}$ & $\mathrm{D}$ & $\mathrm{E}$ & $\mathrm{F}$ & G & $\mathrm{A}^{\prime}$ & $\mathrm{B}^{\prime}$ & $\mathrm{C}^{\prime}$ & $\mathrm{D}^{\prime}$ & $E^{\prime}$ \\
\hline Empty/porous & - & - & 50,245 & - & - & - & 601,188 & 320 & 13,824 & 50,653 & 250,047 & 512,000 \\
\hline Woven & 9,735 & 22,928 & 47,125 & 111,722 & 157,279 & 242,407 & 544,530 & - & 51,223 & 96,851 & 257,270 & 534,040 \\
\hline Overlapped & 13,028 & - & 55,594 & 109,555 & 192,783 & 254,711 & - & 35,395 & 66,124 & 120,586 & 235,053 & - \\
\hline
\end{tabular}

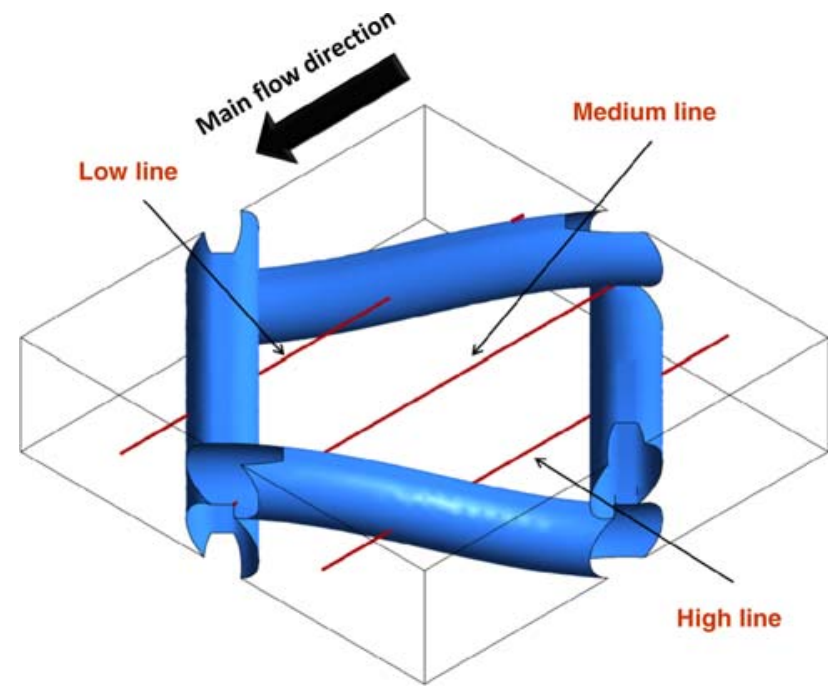

Fig. 7. Monitoring lines parallel to the main flow direction.

discussed in the next section, the results obtained using a tetrahedral/hexahedral hybrid grid with more than $75 \%$ of the computational volume occupied by hexahedral volumes showed a lower grid dependence than those obtained by a purely tetrahedral grid. As a consequence, grids having $\sim 250,000$ volumes were used for all the channel types and the grid topologies, thus resulting into a maximum discrepancy lower than $3 \%$.

\subsection{Grid topology analysis}

Fig. 10 presented in the former section shows also large differences between the results from tetrahedral and hybrid grids: in particular, the average velocity values provided by the tetrahedral grids approach a constant value with a larger number of volumes.

The influence of the grid topology on results was addressed also for the simple empty channel geometry. Fig. 11 shows the pressure drop as a function of the liquid flow rate for the two grid topologies and significant differences can be observed. In particular,
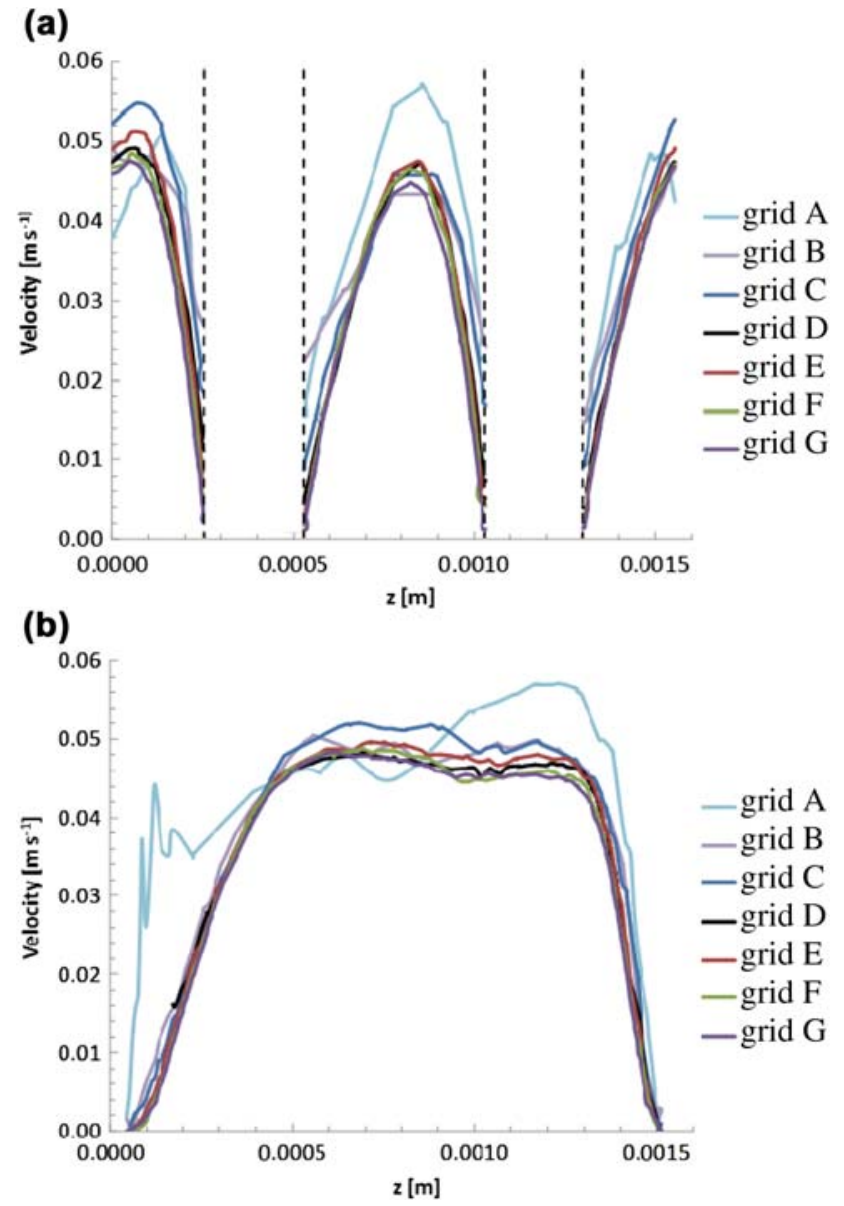

Fig. 8. Velocity profiles $(\Delta \mathrm{P} / 1=0.1 \mathrm{bar} / \mathrm{m})$ with different tetrahedral computational grids for the case of the woven spacer-filled channel. (a) Along the "high" line and (b) along the "medium" line.

the results obtained by employing the hexahedral grids $C^{\prime}$ and $D^{\prime} \quad(\sim 50,000$ and $\sim 250,000$ volumes, respectively) perfectly match the exact pressure drops calculated using the Hagen-Poiseuille equation with average differences of $0.03 \%$ probably due to the lateral extent of the computational domain, finite in the CFD simulations and infinite in the Hagen-Poiseuille solution. 
(a)

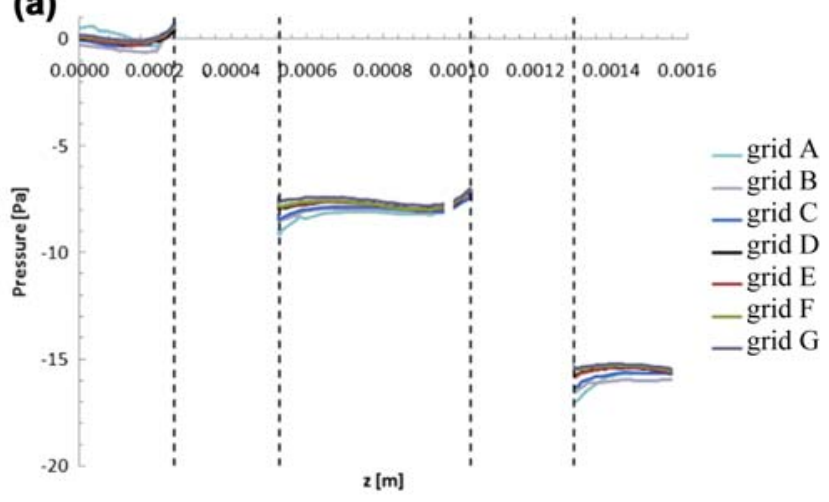

(b)

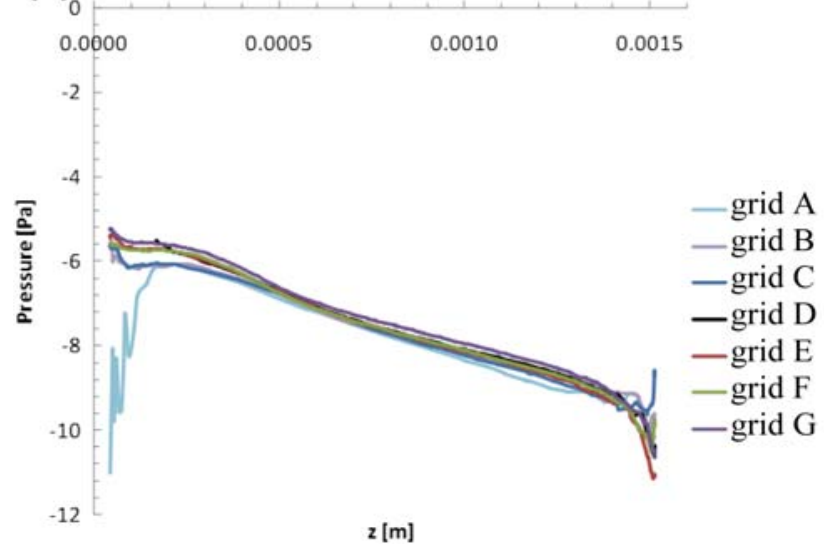

Fig. 9. Pressure profiles $(\Delta \mathrm{P} / 1=0.1 \mathrm{bar} / \mathrm{m})$ with different tetrahedral computational grids for the case of the woven spacer-filled channel. (a) Along the "high" line and (b) along the "medium" line.

Using a tetrahedral grid $C$, an average overestimation of $14.0 \%$ was obtained (this difference increases with the flow rate). Even when a much finer (grid G, i.e. 600,000 volumes) tetrahedral grid was employed, non-negligible differences were obtained $(+6.8 \%)$ in $\Delta \mathrm{P} / \mathrm{l}$. Moreover, since the flow regime is laminar and self-similar in the empty channel, the $\Delta \mathrm{P} / \mathrm{l}$ vs. $Q$ trends are expected to be perfectly linear as predicted by the Hagen-Poiseuille equation. Only the trend obtained by using the hexahedral grid is linear, while the tetrahedral grid results are not well aligned. This finding is in accordance with the already discussed results of Fig. 10: the discretization of the computational domain by tetrahedral requires much finer meshes and consequent much larger simulation times.

Similar considerations can be inferred by the observation of the $\Delta \mathrm{P} / 1$ vs. $Q$ trends relevant to the two spacer-filled channel configurations (Fig. 12). Also in this case, there is a non-negligible discrepancy between the results relevant to the two grid topologies.
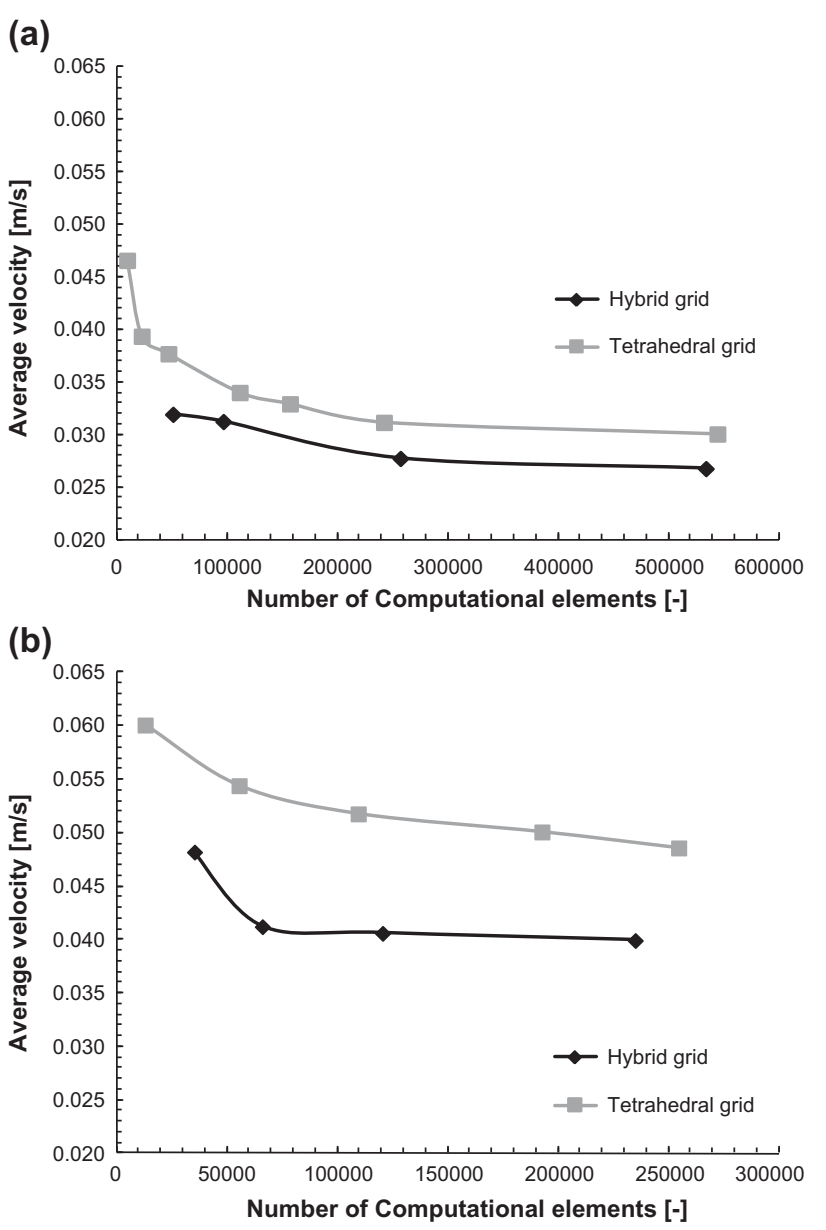

Fig. 10. Dependence of average velocity on mesh size. (a) Woven spacer-filled channel and (b) overlapped spacerfilled channel.

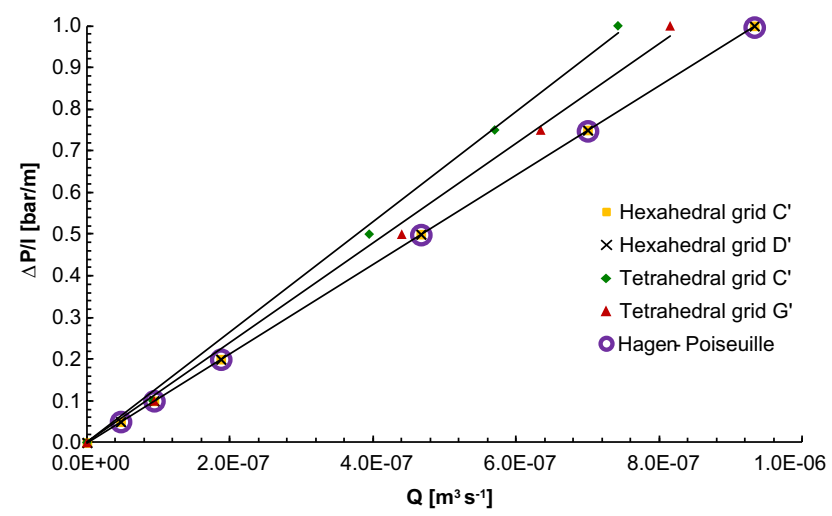

Fig. 11. Comparison among results obtained using hexahedral and tetrahedral grids for the empty channel.

All these results are in accordance with the findings of Molyneux and Bose [13] and Oguntade et al. [14]: although tetrahedral grids are much more easily 


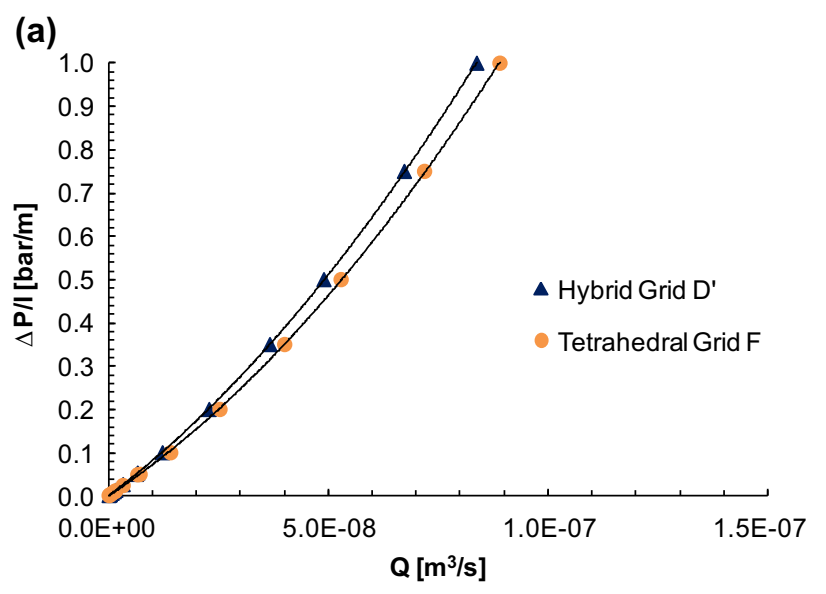

(b)

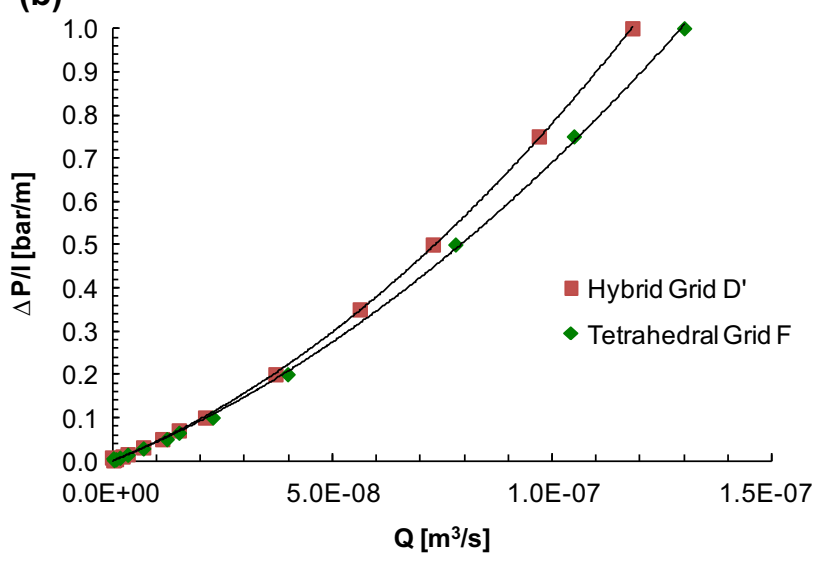

Fig. 12. Comparison among results obtained using hexahedral and tetrahedral grids having $\sim 250,000$ volumes for (a) woven spacer and (b) overlapped spacer-filled channel configurations.

implemented in CFD codes and suitable for complex geometries, a close matching between experimental and predicted results can be obtained only by employing a hexahedral mesh or, at least, a hybrid grid with the highest possible fraction of hexahedral volumes.

\subsection{Transient vs. steady state simulations}

Both steady state and time dependent simulations were performed for the case of the unit cell approach.

Time dependent simulations were carried out by adopting a time step length being in accordance with the Courant criterion:

$\mathrm{Co}=\frac{u \Delta t}{\Delta l}<1$

where Co is the Courant number and $\Delta l$ is the smallest grid size. Five iterations were found to be sufficient to allow residuals to settle before the next time step. A total simulation time of $1 \mathrm{~s}$ allowed steady state conditions to be achieved for all the cases investigated. Pressure and velocity values equal to zero were chosen as initial condition.

In steady state simulations, the number of iterations was chosen for all the cases investigated so that the root-mean-square normalized residuals of pressure and velocity fell below $1 \times 10^{-6}$.

The comparison between the two simulation types was made for the case of an imposed pressure drop of $0.1 \mathrm{bar} / \mathrm{m}$, i.e. the upper limit of the operating range of a typical ED/RED apparatus.

A perfect agreement between results obtained with steady state and transient simulations was obtained for all channel configurations. Such findings fully justify the choice of adopting the steady state simulations in view of the corresponding computational savings.

\subsection{Definition of $\Delta \mathrm{P} / 1$ for Unit Cell approach}

The Unit Cell approach requires i) to impose periodic velocity boundary conditions on the in- and outsurfaces along the main flow direction and ii) to set a pressure drop in the same direction in order to allow the fluid to move within the domain and a corresponding flow field to be calculated.

There are two different ways to impose the pressure drop:

- the explicit imposition of two different pressures at the inlet and outlet surfaces;

- the inclusion of a force per unit volume $F$ in the right hand side of the streamwise $(\mathrm{Z})$ momentum equation as previously anticipated in Section 3 .

According to the momentum equation, the material derivative of the velocity depends on the viscous stresses, the pressure gradient, and the volume forces. These latter two terms are not distinguishable by the code from a mathematical point of view, therefore it is possible to impose either a pressure gradient and no volume forces or vice versa to get the same flow field. No appreciable difference was found. Thus, only unit cell simulations along with the imposition of a pressure difference between the inlet and the outlet surfaces will be discussed in the following.

\section{Results and discussion}

In the present section, the results relevant to each channel configuration (empty, porous, and spacer filled) will be first described separately. Such results concern: 
- the relation between flow rate and pressure drops along the channel;

- the drag coefficient dependence on the Reynolds number;

- the fluid flow field.

Finally, a comparison between the various configurations will be provided.

\subsection{Fluid flow regime and flow field analysis}

\subsubsection{Empty channel}

The empty channel represents the simplest geometrical configuration: it is able to guarantee the lowest pressure drops since the fluid does not encounter any obstacle within the channel. On the other hand, the absence of obstacles causes a scarce fluid mixing thus resulting in larger concentration polarization effects. The relation between liquid flow rate and pressure drops for the empty channel is perfectly linear as expected for all the range of pressure drops investigated (up to a Re number of about 1,500).

Clearly, the friction coefficient $f$ vs. Reynolds number was found to be proportional to $1 / \operatorname{Re}$ in all the flow rate range investigated and solutions were perfectly steady thus confirming that the flow regime is laminar.

In addition, all the velocity vectors plotted on planes parallel to the liquid main flow direction, not shown here for brevity reasons, show the parabolic trend typical of laminar regimes in empty channels or tubes. Finally, the flow field is perfectly self-similar for all the test cases investigated as expected (Figs. 15 (a), 16(a), and 17(a)).

\subsubsection{Spacer-filled channel}

The adoption of a spacer-filled channel configuration may constitute a good compromise between the features exhibited by the empty channel and a low porosity porous medium filled channel: the presence of a spacer may lead both to a good mixing (i.e. low polarization concentration effects) and acceptable pressure drops.

The relation between water flow rate and pressure drops is similar for both spacer types and is not linear (Fig. 13). The vertical dashed line indicates the upper limit of the typical operating range for ED and RED processes (i.e. pressure losses of $0.1 \mathrm{bar} / \mathrm{m}$ ). At flow rates lower than the former limit, the trend of $\Delta \mathrm{P} / 1$ vs. $Q$ is perfectly linear as a close observation of Fig. 13 shows.
Although Fig. 13 shows a nonlinear behavior, the fluid flow regime is however laminar for both the spacer-filled channel configurations, since the corresponding solutions were found to be perfectly steady.

CFD simulations at higher flow rates were carried out aiming at (1) approximately identifying the minimum Reynolds number at which solutions started exhibiting a chaotic behavior with time and (2) characterizing the two spacers up to high Reynolds numbers.

This cut-off Reynolds number was found to be about 500 for both the two spacers. Simulations relevant to Reynolds number lower than this value were performed without employing any turbulence model, while the cases relevant to higher Reynolds number were simulated by employing the $k-\omega$ SST as shown Fig. 14. In this figure, the friction factor $f$ vs. Re curves are presented: the results which are on the left of the vertical dash-dot line (i.e. Reynolds number corresponding to the pressure losses of $1 \mathrm{bar} / \mathrm{m}$ ) are corresponding to the simulations already presented in
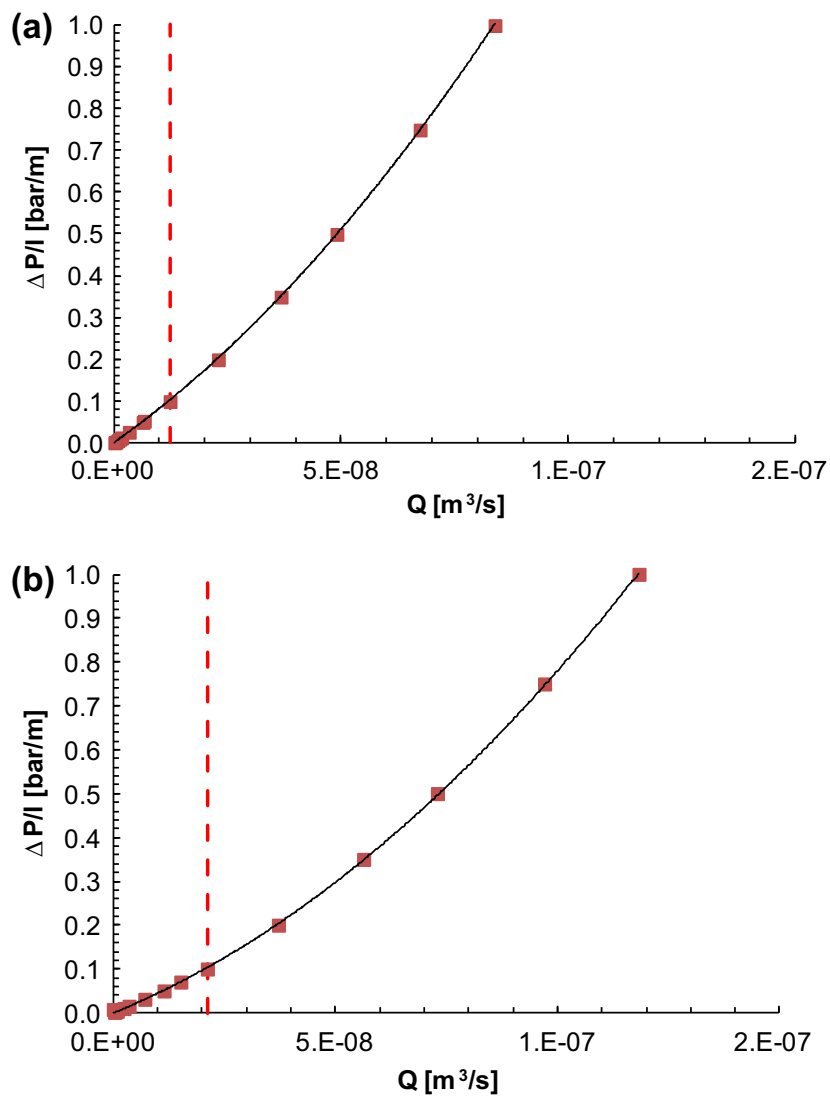

Fig. 13. Pressure drops as a function of the fluid flow rate: (a) woven spacer-filled channel and (b) overlapped spacerfilled channel. The dashed line indicates the upper limit of the typical operating range for ED and RED processes. 

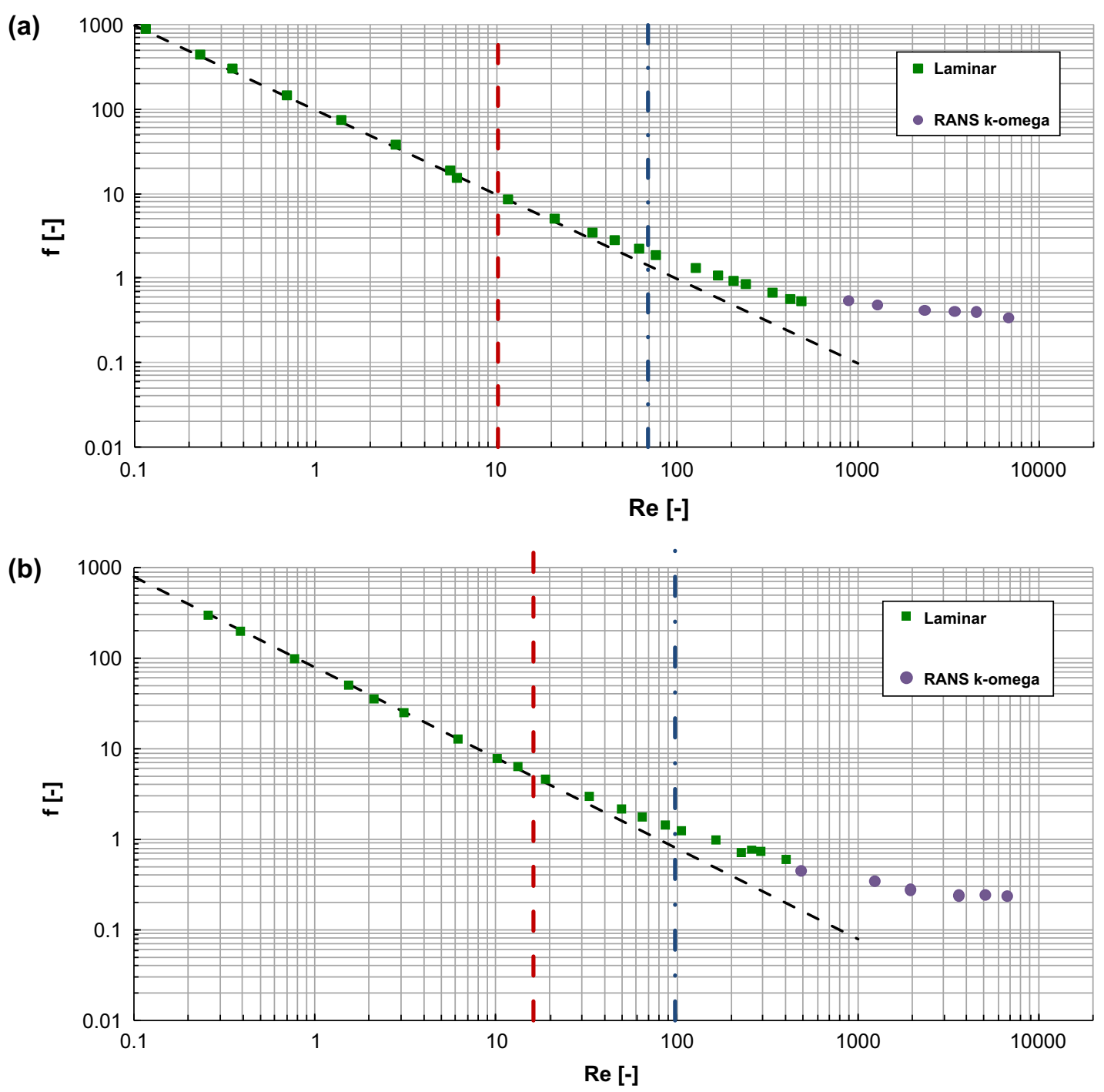

Fig. 14. Drag coefficient as a function of the Reynolds number for the case of the spacer-filled channels: (a) woven spacerfilled channel and (b) overlapped spacer-filled channel. The dashed vertical line indicates the typical operating range for $\mathrm{ED}$ and RED processes. The dash-dot vertical line indicates the Reynolds number corresponding to a pressure drop $\Delta \mathrm{P} / 1$ $=1 \mathrm{bar} / \mathrm{m}$.

Fig. 13. A slope equal to -1 of the $f$ vs. Re curves was found for all the cases relevant to the operating range of ED/RED (i.e. Reynolds numbers lower than the one corresponding to the dashed vertical line), while a nonlinear behavior can be observed at higher Reynolds numbers, as already seen in Fig. 13. The results relevant to the simulations carried out with the $k-\omega$ SST turbulence model show that at Reynolds numbers higher than about $2,000, f$ starts to become constant.

It is worth noting that when the channel is filled with a net spacer, the achievement of turbulent conditions occurs at a Reynolds number (i.e. about 500, see Fig. 14) lower than that relevant to the empty channel (i.e. $>1,500$ ). This occurence was already observed by other authors for the case of spacer-filled channels designed for applications other than RED [24] and it is also a typical occurence of packed beds $[25,26]$.

The nonlinear dependence of $\Delta \mathrm{P} / 1$ on $Q$ within a laminar regime is due to the presence of the spacer, which forces the fluid to move more chaotically thus resulting in a flow field which is not self-similar as the fluid flow rate increases.

In Fig. 15(b,c), the velocity module contours plotted on different $\mathrm{YZ}$ planes (which are parallel to the fluid flow) are shown for the two different spacerfilled channels at three different imposed pressure drops (i.e. $0.01,0.1$, and $1 \mathrm{bar} / \mathrm{m}$ ). 


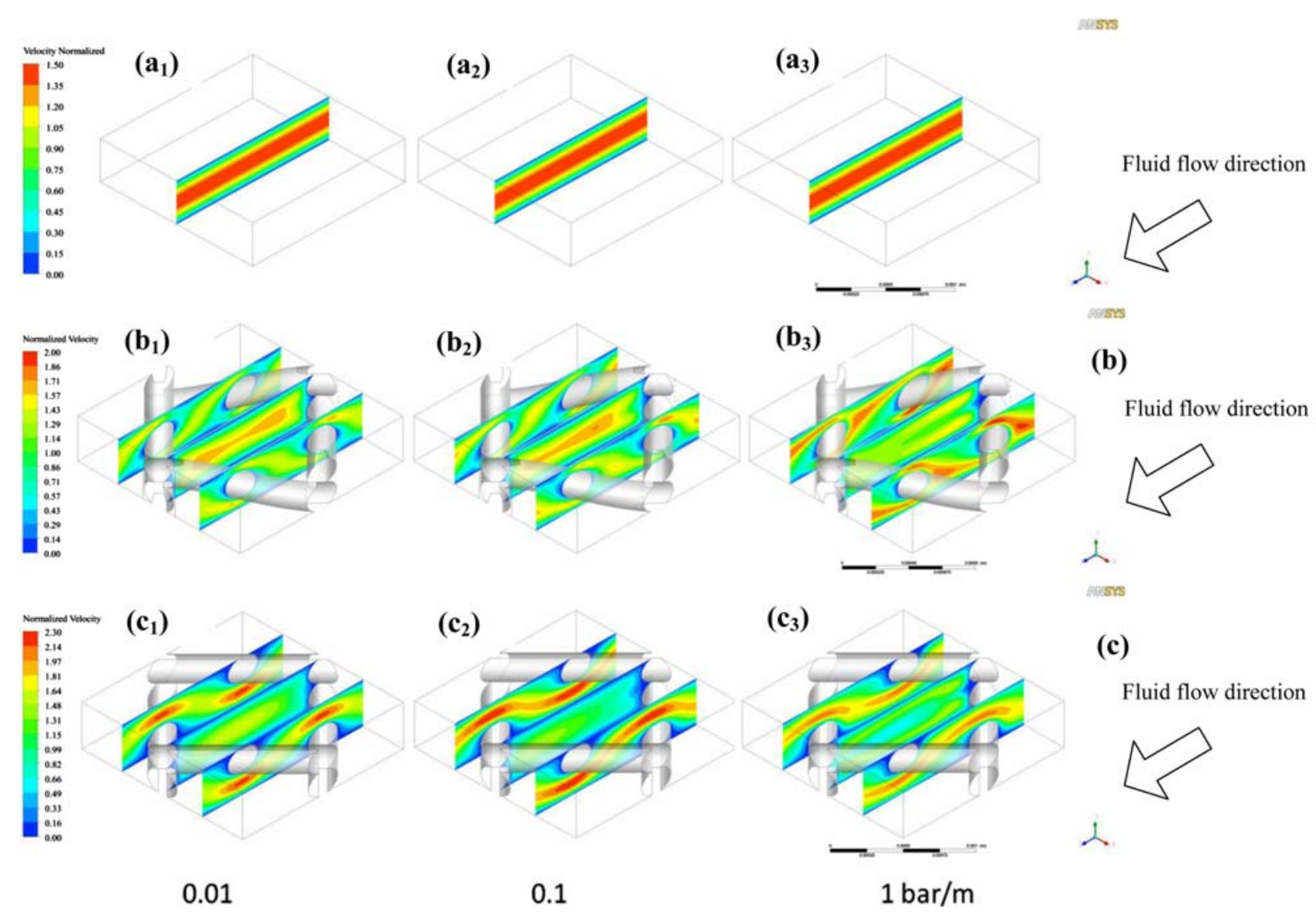

Fig. 15. Contour plots of the velocity module on different YZ-planes at three different imposed pressure drops for the case of: (a) empty channel, (b) woven spacer-filled channel, and (c) overlapped spacer-filled channel.

As far as the woven spacer-filled channel is concerned, as it can be seen in the lateral (high and low) contours plots of Fig. 15(b), the velocity increases when the fluid pass through the section reduced because of the filament presence. Notably, since the filaments have a sinusoidal-like shape, the fluid may move both above and below the filament itself. By comparing the three countours (b) of Fig. 15, it can be observed that the corresponding flow fields are not self-similar as previously hyphotized: in particular, at $\Delta \mathrm{P} / \mathrm{l}=0.01 \mathrm{bar} / \mathrm{m}$ and $0.1 \mathrm{bar} / \mathrm{m}$ (i.e. Fig. $15\left(\mathrm{~b}_{1}\right)$ and $\left.\left(b_{2}\right)\right)$ high velocities appear to be located in the central part of the unit cell, while at $\Delta \mathrm{P} / \mathrm{l}=1 \mathrm{bar} / \mathrm{m}$ (Fig. 15 $\left.\left(b_{3}\right)\right)$ in the same location the fluid moves more slowly with respect to the lateral planes.

A less pronounced lack in self-similarity is also observable for the case of the channel filled with the overlapped spacer (Fig. 15(c)). Moreover, as a difference from the woven spacer-filled channel, here the fluid moves either above the low filaments or below the high filaments as expected.
The above considerations can be also inferred from Fig. 16(b,c) and Fig. 17(b,c), where velocity module contours on different planes are shown.

In particular, contours plots of the velocity module on different XY-planes at the same three imposed pressure drops are shown in Fig. 16. As it can be seen, the flow within the empty channel is perfectly selfsimilar while the flow in the woven spacer-filled channel exhibits the most evident lack in self-similarity. Also, as the imposed pressure drops increase, the maximum values of velocity are not located in the central part of the channel (i.e. the part free of filaments), but close to the filaments because of the restricted passage area.

In Fig. 17, contours of the velocity module are plotted on the central $\mathrm{XZ}$ plane. This figure shows an upstream/downstream symmetric flow field for both the two spacer-filled channels at the lowest pressure drop, thus suggesting the existence of a creeping flow. Conversely, the flow field becomes asymmetric as the imposed pressure drops increase. 

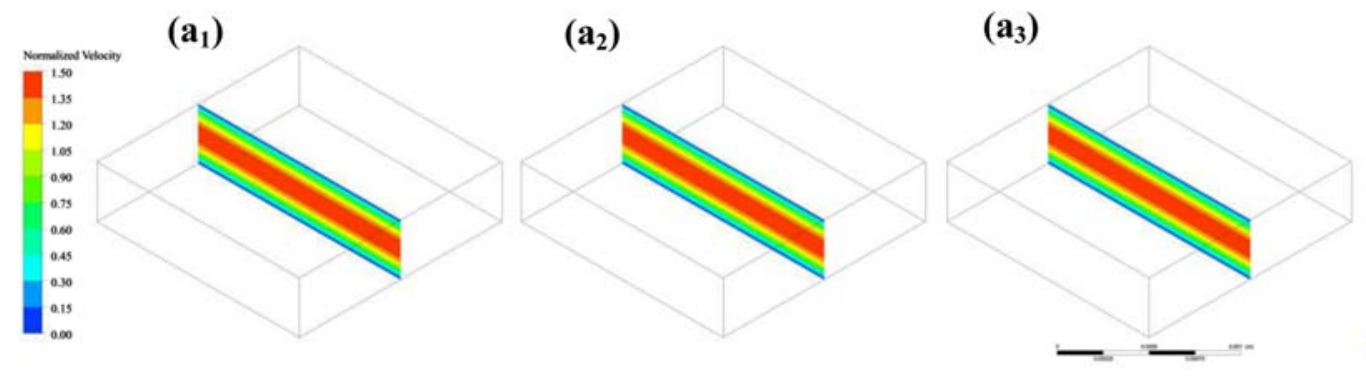

(a)

$\left(\mathbf{b}_{1}\right)$

$\left(\mathbf{b}_{2}\right)$

(b)
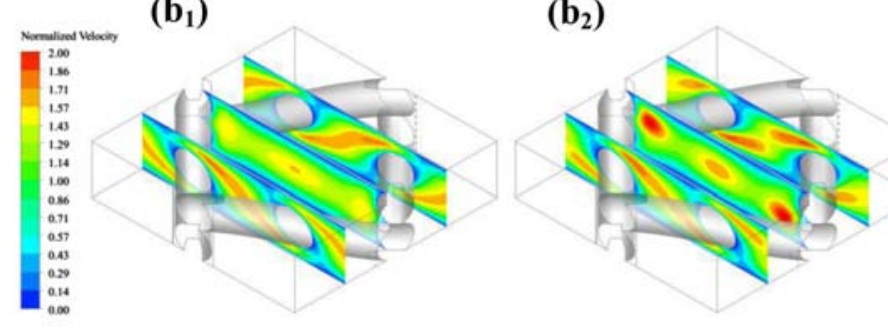

(b)

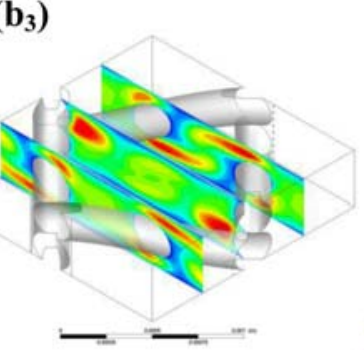

(b)

Fluid flow direction

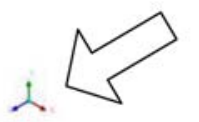

$\left(c_{1}\right)$

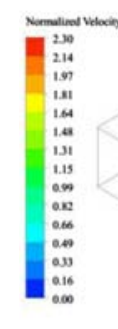

$\left(c_{2}\right)$

(c)

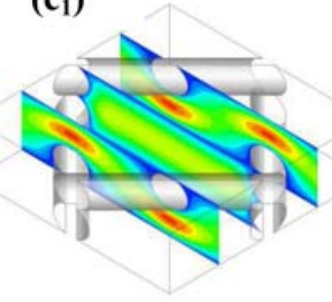

0.01

0.1

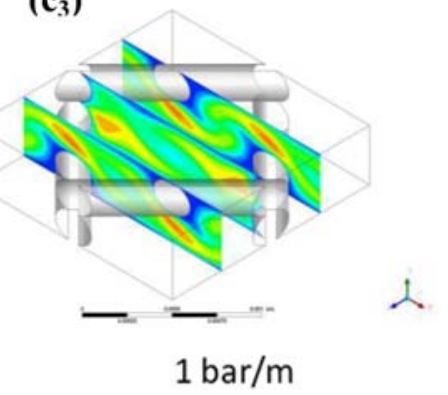

(c)

Fluid flow direction

Fluid flow direction

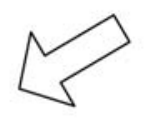

Fig. 16. Contour plots of the velocity module on different XY-planes at three different imposed pressure drops for the case of: (a) empty channel, (b) woven spacer-filled channel, and (c) overlapped spacer-filled channel.

The existence of a creeping condition at the lowest $\Delta \mathrm{P} / 1$ of $0.01 \mathrm{bar} / \mathrm{m}$ is confirmed by the observation of Fig. 18, where planar projections of trajectories on a YZ-plane are shown: at this low imposed pressure drop the trajectories seem to follow quite well the filament shape for both the two spacer-filled channels. As the imposed $\Delta \mathrm{P} / 1$ increases, all the trajectories show an earlier detachment from the filament as well as a larger woke region.

Finally, Fig. 19 shows normalized velocity vectors on the central XZ-plane at two different imposed pressure drops. For both the two spacers, as the lowest imposed pressure drops, the flow pattern is planar in the whole region but in the proximities of filaments.

Also, their shape is followed by the velocity vectors thus confirming the existing of creeping conditions. Conversely, very strong vertical component as well as a general chaotic behavior are observable at the highest pressure drops as a mark of the achievement of turbulent conditions within the spacer-filled channel domain.

\subsubsection{Porous channel}

The results obtained for the case of the channel filled with the purposely manufactured porous medium are similar to those relevant to the empty channel.

The fluid flow regime is laminar. As a matter of fact, the relation of the flow rate with the pressure drops was found to be linear, but with a slope higher than that relevant to the spacer-less channel as expected.

The present configuration has fluid flow characteristics being contrary to that of the empty channel: on the one hand, the fluid flowing through the porous medium is likely to be subjected to a strong mixing and to negligible concentration polarization effects consequently, on the other hand, at a given flow rate the employed porous medium gives rise to pressure drops along the channel much higher than those obtainable for the case of an empty channel (Fig. 20). 

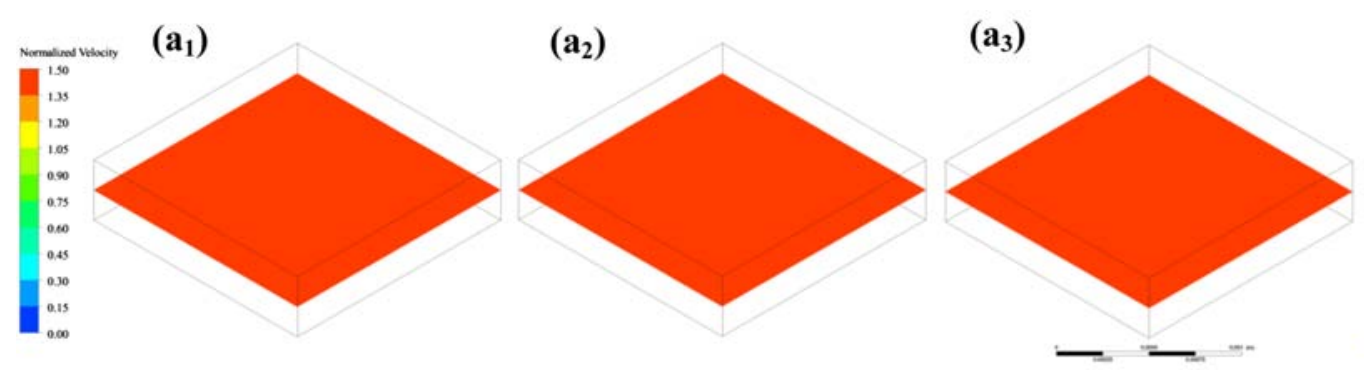

ตถ⿻上丨ร

$\left(\mathbf{b}_{1}\right)$

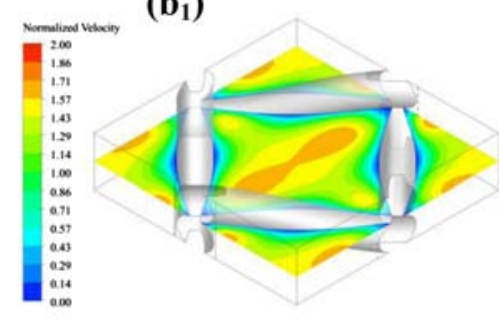

$\left(c_{1}\right)$

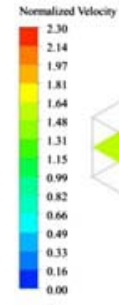

$\left(\mathbf{b}_{2}\right)$

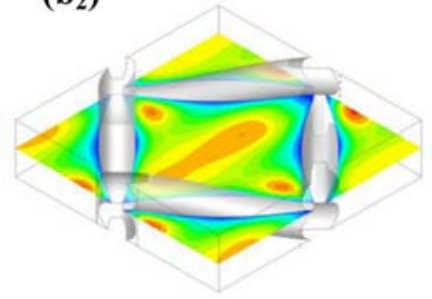

$\left(c_{2}\right)$ $\left(\mathbf{b}_{3}\right)$

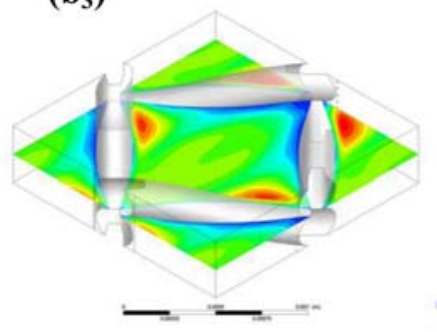

(c)

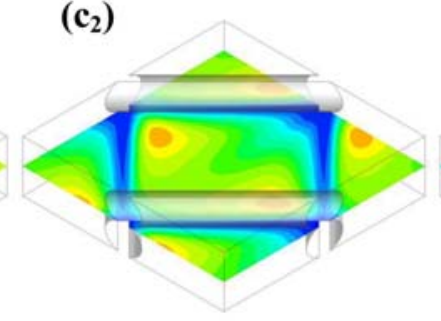

0.1

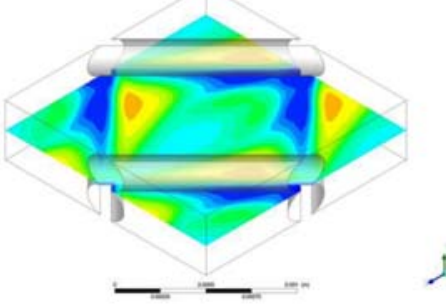

$1 \mathrm{bar} / \mathrm{m}$

(b)

Fluid flow direction

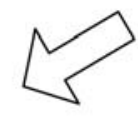

人

(c)

Fluid flow direction

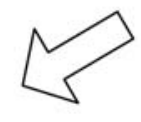

0.01

Fig. 17. Contour plots of the velocity module on the central XZ-plane at three different imposed pressure drops for the case of: (a) empty channel, (b) woven spacer-filled channel, and (c) overlapped spacer-filled channel.

Notably, all the results found for the porous medium filled channel should be considered specifically relevant to the porous medium investigated here: different results will be obtained if a porous medium with different characteristics is employed.

\subsection{Comparison among channel configurations}

All the results presented so far concerning the relation between the flow rates and the pressure drops along the channel for all the investigated configurations are shown in the same figure (Fig. 20) for comparison purposes. Clearly, the empty channel guarantees the lowest energy consumption at a given flow rate. Conversely, although the channel filled with the commercial porous medium is likely to provide the best fluid mixing, on the other hand it generates the highest pressure drops along the channel. The presence of the spacer is known to reduce the concentration polarization phenomenon [27] in turn of increased pressure drops. As it can be seen in Fig. 20, the adoption of a spacer-filled channel may constitute a good compromise between these opposing aspects.

The Fig. 21 shows the velocity value as a function of the $\mathrm{Z}$ coordinate (i.e. the coordinate parallel to the fluid flow direction) in the two different monitoring lines high and medium for the case of an imposed $\Delta \mathrm{P} /$ $1=0.1 \mathrm{bar} / \mathrm{m}$. As previously discussed, the results relevant to the low line are mirror-like to those relevant to the high line and are not shown for brevity. By observing this Fig. 21, it can be noted that the velocity relevant to the empty channel are 7-8 times larger than those relevant to the spacer-filled channels, thus suggesting again that an empty channel can guarantee large savings of pumping costs. Conversely, the employment of the porous medium provides very low velocities along the channel as expected. As far as the spacer-filled channels are concerned, the velocity vs. Z trend is very similar for the two cases, but the velocity within an overlapped spacer-filled channel is about $50 \%$ larger than those relevant to the woven spacerfilled channel along the line high. Conversely, there 

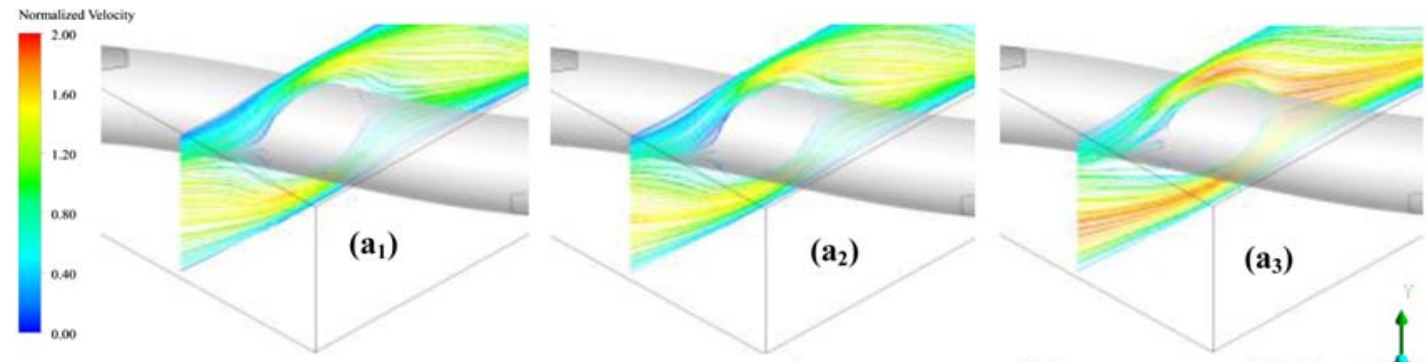

$\left(\mathbf{a}_{2}\right)$

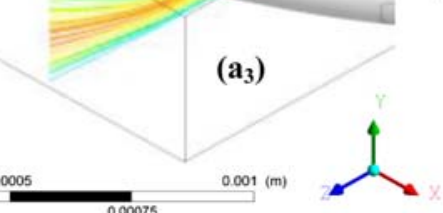

(a)

Fluid flow direction
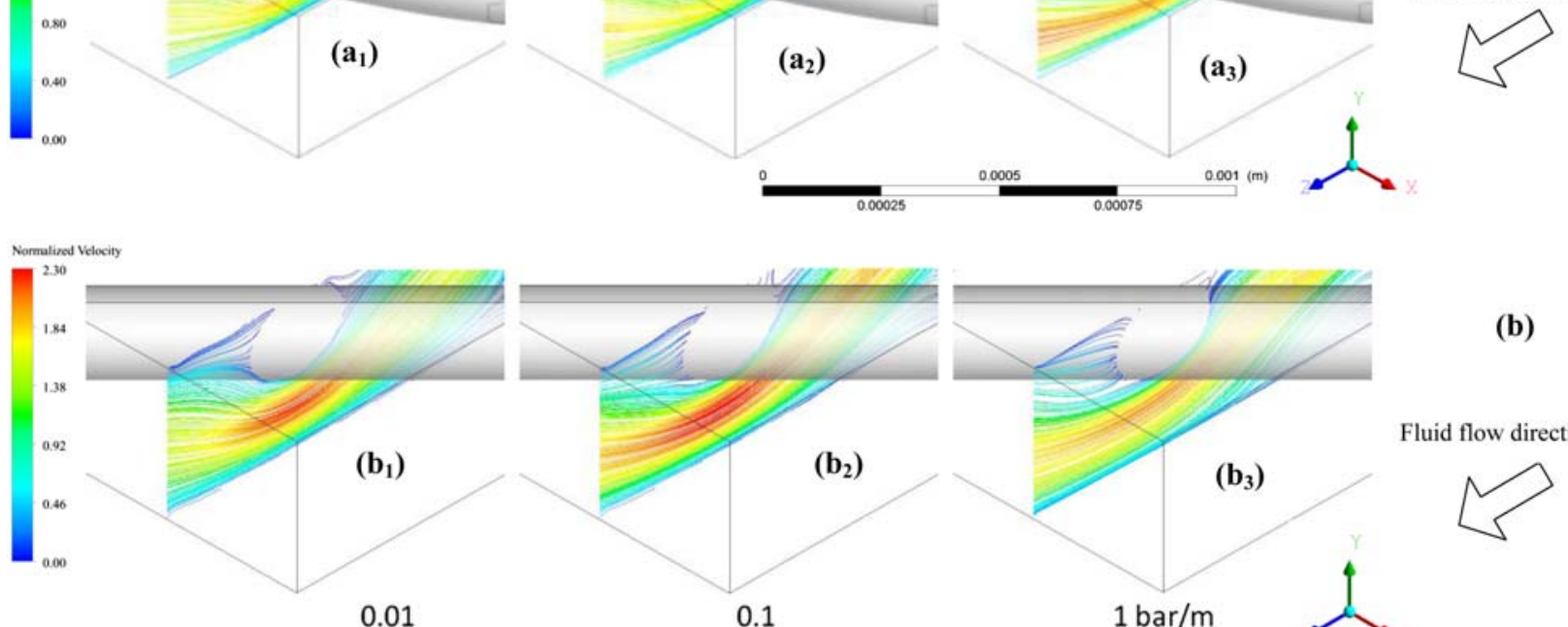

(b)

Fluid flow direction

0.01

0.1
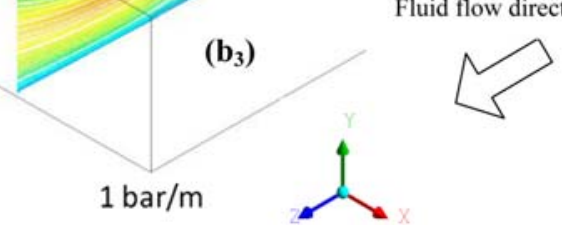

Fig. 18. Zoom of planar projections of trajectories (colored according to the normalized velocity module) on a YZ-plane at three different imposed pressure drops for the case of: (a) woven spacer-filled channel and (b) overlapped spacer-filled channel.

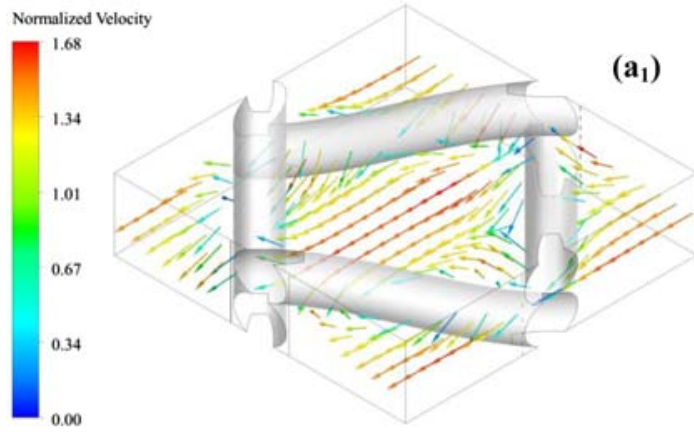

$0.01 \mathrm{bar} / \mathrm{m}$

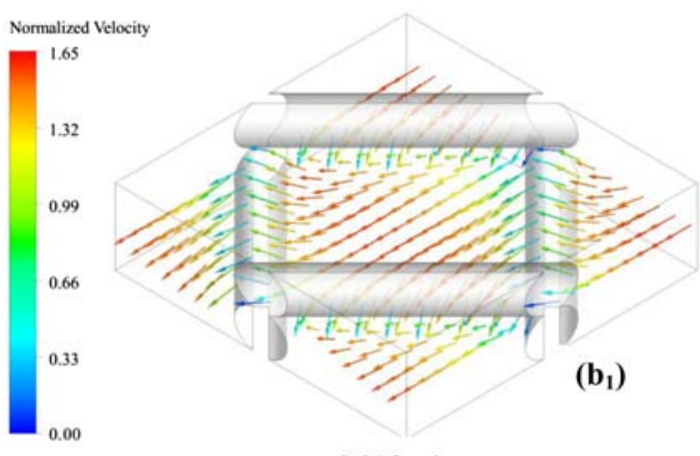

$0.01 \mathrm{bar} / \mathrm{m}$

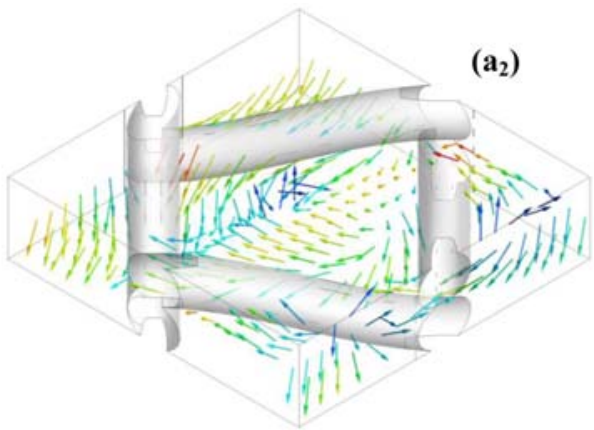

$1000 \mathrm{bar} / \mathrm{m}$

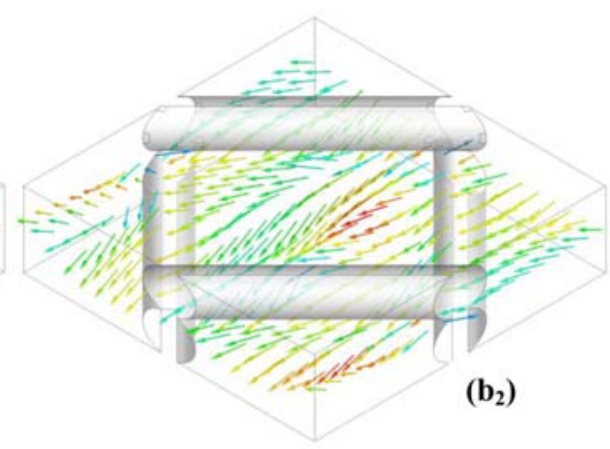

$1000 \mathrm{bar} / \mathrm{m}$ (a)

Fluid flow direction<smiles>C1CCCCC1</smiles>

Fig. 19. Normalized velocity vectors (colored according to the normalized velocity module) on the central XZ-plane at two different imposed pressure drops for the case of: (a) woven spacer0filled channel and (b) overlapped spacer-filled channel. 


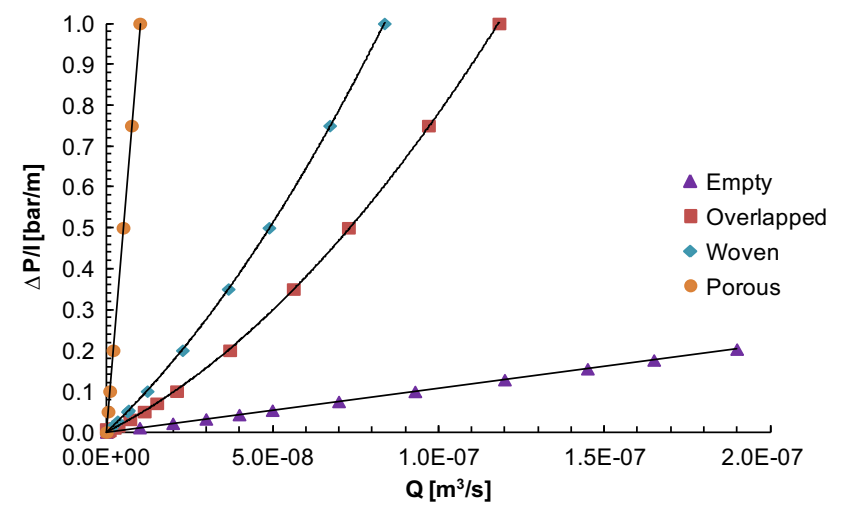

Fig. 20. Pressure drops as a function of the fluid flow rate: comparison among the different channel configurations. are no significant differences in the velocities values within the two spacer-filled channels along the medium line. Notably, these two trends along the medium line are not identical allegedly because of the different filament geometries which influences the flow pattern in the central part of the unit cell (where the medium line is located).

The variation of the pressure along the channels for the two monitoring lines is shown in Fig. 22 at the same imposed $\Delta \mathrm{P} / 1=0.1 \mathrm{bar} / \mathrm{m}$. Clearly, the trend is identical for the case of the empty and the porous medium filled channel since no obstacles are included in the computational domain. A different decreasing trend was found for the case of a channel including spacers. As concerns the medium monitoring line (i.e. the central part of the unit cell where no filaments are
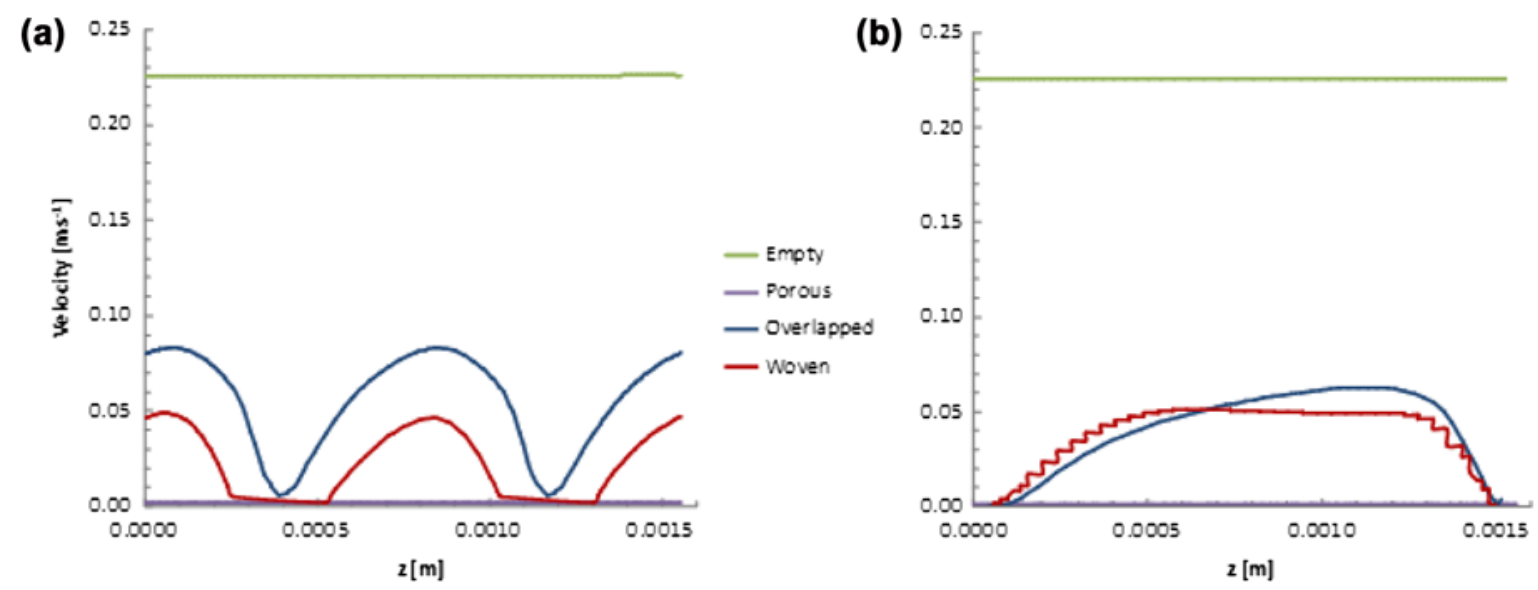

Fig. 21. Velocity profiles along the Z-coordinate at an imposed $\Delta \mathrm{P} / \mathrm{l}=0.1 \mathrm{bar} / \mathrm{m}$ : comparison among the different channel configurations. (a) High line and (b) Medium line.
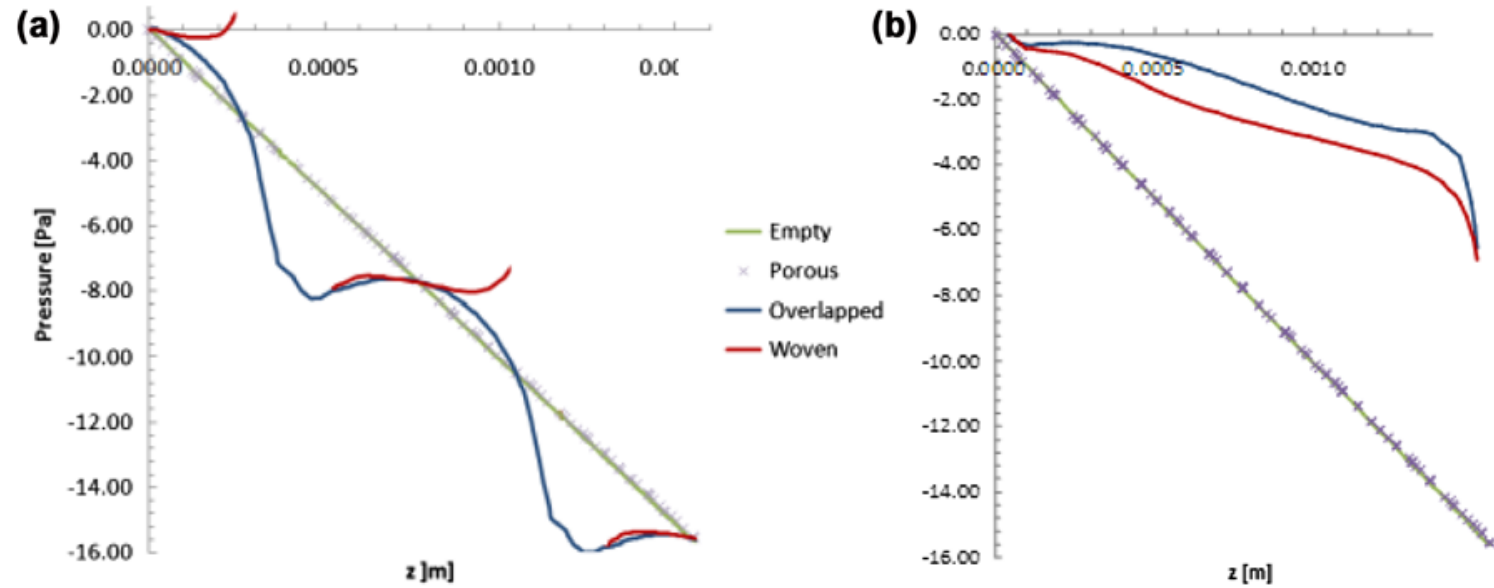

Fig. 22. Pressure profiles along the Z-coordinate at an imposed $\Delta \mathrm{P} / \mathrm{l}=0.1 \mathrm{bar} / \mathrm{m}$ : comparison among the different channel configurations. (a) High line and (b) Medium line. 
present), the two spacer-filled channels exhibit a similar monotonic behavior (Fig. 22(b)). Conversely, a different step-like trend is observable along the high line (Fig. 22(a)): the difference between the two spacerfilled channel cases is due to the relative position between the monitoring high line and the filaments. In the case of the overlapped filled channel, the monitoring line is practically tangent to the filaments, while in the case of the woven filled channel, the line completely intersects the filaments.

\section{Conclusions and future remarks}

In the present work, different channels for ED and RED applications were investigated by means of CFD along with the Unit Cell approach [16,15]. The relation between the pressure drops and the fluid flow rate was predicted for some different channel configurations: an empty channel, a channel filled with a purposely manufactured fiber structure porous spacer, a channel provided with a commercial spacer made of woven perpendicular filaments, and a channel filled with a spacer made of overlapped perpendicular filaments. A sensitivity analysis including among the others the effect of grid size and topology was performed. In this regard, completely hexahedral grids or hybrid grids mainly composed of hexahedral volumes were found to be more reliable and less computational demanding than the tetrahedral ones.

The $k-\omega$ SST turbulence model was employed to investigate the net spacer-filled channels up to large Reynolds numbers, where turbulent conditions are achieved.

As far as the dependence of the pressure drops on the flow rate is concerned, the empty channel was found to guarantee the lowest pressure drops at a given fluid flow rate, while the woven spacerfilled channel was found to provide pumping costs larger than those relevant to the overlapped spacerfilled channel and to the empty channel. The pressure losses caused by the channel filled with the fiber porous medium even at low flow rates were very high, thus suggesting that this specific configuration may be unsuitable for the real process. Nevertheless, the proper attention should be paid to find the features a porous spacer should have to provide similar to better performance with respect to the common net spacer.

The findings concerning the flow field analysis within the two net spacer-filled channels suggest that, even at low $\operatorname{Re}$ and under laminar regime, the flow is quite complex and characterized by a lack in self- similarity as the flow rate increases. This implies that attention should be paid in formulating and managing correlation for these systems.

Notably, data concerning the relation between the liquid flow rate and the pressure drops are not sufficient to establish which is the more effective channel configuration. Such data should be coupled with other data regarding concentration polarization phenomena. As a consequence, future works will address the transport of ionic species along the channels as well as across the membranes in order to predict the concentration polarization factor. The coupling between data concerning the pressure drops (presented in this work) and future data regarding the concentration polarization factor for each different configurations will be useful to guide the design of apparatuses devoted to ED and RED operations.

\begin{tabular}{|c|c|}
\hline \multicolumn{2}{|c|}{ Notations } \\
\hline Co & Courant number (-) \\
\hline$d_{\mathrm{h}}$ & hydraulic diameter (m) \\
\hline$f$ & friction coefficient $(-)$ \\
\hline$F$ & force for volume unit $\left(\mathrm{N} \mathrm{m}^{-3}\right)$ \\
\hline$\Delta l$ & smallest grid size (m) \\
\hline$k$ & turbulent kinetic energy $\left(\mathrm{m}^{2} \mathrm{~s}^{-2}\right)$ \\
\hline$k_{\text {Darcy }}$ & Darcy's constant $\left(\mathrm{m}^{2}\right)$ \\
\hline$P$ & pressure $(\mathrm{Pa})$ \\
\hline$\Delta \mathrm{P} / 1$ & pressure drops for length unit $\left(\mathrm{Pa} \mathrm{m}^{-1}\right)$ \\
\hline$Q$ & flow rate $\left(\mathrm{m}^{3} \mathrm{~s}^{-1}\right)$ \\
\hline $\operatorname{Re}$ & Reynolds number (-) \\
\hline$u$ & velocity $\left(\mathrm{m} \mathrm{s}^{-1}\right)$ \\
\hline $\bar{u}$ & average velocity within the unit cell $\left(\mathrm{m} \mathrm{s}^{-1}\right)$ \\
\hline$\Delta t$ & time step length (s) \\
\hline \multicolumn{2}{|c|}{ Greek letters } \\
\hline$\varepsilon$ & turbulent dissipation $\left(\mathrm{W} \mathrm{kg}^{-1}\right)$ \\
\hline$\mu$ & viscosity (Pa s) \\
\hline$v$ & kinematic viscosity $\left(\mathrm{m}^{2} \mathrm{~s}^{-1}\right)$ \\
\hline$\rho$ & density $\left(\mathrm{kg} \mathrm{m}^{-3}\right)$ \\
\hline$\omega$ & turbulent frequency $(\mathrm{Hz})$ \\
\hline
\end{tabular}

\section{Acknowledgments}

This work has been performed within the REAPower (RED Alternative Power production) project funded by the EU-FP7 programme (Project Number: 256736) - website www.reapower.eu. Authors acknowledge Dr. E. Brauns and VITO for their precious suggestions concerning the adoption of the porous spacer. NTT is also acknowledged for porous spacer manufacturing and testing. 


\section{References}

[1] R.E. Lacey, Energy by reverse electrodialysis, Ocean Eng. 7 (1980) 1-47.

[2] J.W. Post, H.V.M. Hamelers, C.J.N. Buisman, Energy recovery from controlled mixing salt and fresh water with a reverse electrodialysis system, Environ. Sci. Tech. 42 (2008) 5785-5790.

[3] S. Loeb, Production of energy from concentrated brines by pressure retarded osmosis. 1. Preliminary technical and economic correlations, J. Membr. Sci. 1 (1976) 49-63.

[4] W. Finley, E. Pscheidt, Hydrocratic generator. US Patent 6 313545 B1 (2001).

[5] M. Olsson, G.L. Wick, J.D. Isaacs, Salinity gradient powerutilizing vapor-pressure differences, Science 206 (1979) $452-454$.

[6] J. Veerman, M. Saakes, S.J. Metz, G.J. Harmsen, Reverse electrodialysis: Performance of a stack with 50 cells on the mixing of sea and river water, J. Membr. Sci. 327 (2009) 136-144.

[7] G.L. Wick, W.R. Schmitt, Prospects for renewable energy from sea, Mar. Technol. Soc. J. 11 (1977) 16-21.

[8] E. Brauns, Towards a worldwide sustainable and simultaneous large- scale production of renewable energy and potable water through salinity gradient power by combining reversed electrodialysis and solar power, Desalination. 219 (2008) 312-323.

[9] E. Brauns, W. De Wilde, B. Van den Bosch, P. Lens, L. Pinoy, M. Empsten, On the experimental verification of an electrodialysis model for optimal stack configuration design through solver software, Desalination. 249 (2009) 1030-1038.

[10] M.H. Dirkse, W.K.P. van Loon, J.D. Stigter, J.W. Post, J. Veerman, G.P.A. Bot, Extending potential flow modelling of flatsheet geometries as applied in membrane-based systems, J. Membr. Sci. 325 (2008) 537-545.

[11] A.R. Da Costa, A.G. Fane, D.E. Wiley, Spacer characterization and pressure drop modeling in spacer-filled channels for ultra-filtration, J. Membr. Sci. 87 (1994) 79-98.

[12] S.K. Karode, A. Kumar, Flow visualization through spacer filled channels by computational fluid dynamics-I: Pressure drop and shear rate calculations for flat sheet geometry, J. Membr. Sci. 193 (2001) 69-84.

[13] D. Molyneux, N. Bose, Escort tug at large yaw angle: Comparison of CFD predictions with experimental data, Transactions of the Royal Institution of Naval Architects Part B: International Journal of Small Craft Technology 150 (2008) 41-60.
[14] H.I. Oguntade, G.E. Andrews, A. Burns, D. Ingham, M. Pourkashanian, CFD predictions of single row film cooling with inclined holes: Influence of hole outlet geometry, Proceedings of the ASME Turbo Expo 4 (2010) 1371-1385.

[15] Z.X. Yuan, W.Q. Tao, Q.W. Wang, Numerical prediction for laminar forced convection heat trans- fer in parallel plate channels with streamwise periodic rod disturbances, Int. J. Numerical Meth. Fluids 28 (1998) 1371-1387.

[16] Y.-L. Li, K.-L. Tung, CFD simulation of fluid flow through spacer-filled membrane module: Selecting suitable cell types for periodic boundary conditions, Desalination. 233 (2008) 351-358.

[17] E. Brauns, Salinity gradient power by reverse electrodialysis: Effect of model parameters on electrical power output, Desalination 237 (2009) 378-391.

[18] P. Długołęckia, J. Dąbrowska, K. Nijmeijer, M. Wessling, Ion conductive spacers for increased power generation in reverse electrodialysis, J. Membr. Sci. 347 (2010) 101-107.

[19] O. Kedem, Reduction of polarization in electrodialysis by ionconducting spacers, Desalination 16 (1975) 105-118.

[20] E. Korngold, L. Aronov, O. Kedem, Novel ion-exchange spacer for improving electrodialysis I. Reacted spacer, J. Membr. Sci. 138 (1998) 165-170.

[21] H. Strathmann, Electrodialysis, a mature technology with a multitude of new applications, Desalination 264 (2010) $268-288$.

[22] P. Pitò, A. Cipollina, G. Micale, M. Ciofalo, Characterization of spacer-filled membrane distillation modules by thermochromic liquid crystals. XXIX Congresso UIT sulla trasmissione del calore, Turin, Italy. June 20-22 (2011).

[23] F.R. Menter, Two-equation eddy-viscosity turbulence models for engineering applications, AIAA Journal 32(8) (1994) 1598-1605.

[24] V.V. Ranade, A. Kumar, Fluid dynamics of spacer filled rectangular and curvilinear channels, J. Membr. Sci. 271 (2006) $1-15$.

[25] D. Seguin, A. Montillet, J. Comiti, Experimental characterization of flow regimes in various porous media. I. Limit of laminar flow regime, Chem. Eng. Sci. 53(21) (1998) 3751-3761.

[26] D. Seguin, A. Montillet, J. Comiti, F. Huet, Experimental characterization of flow regimes in various porous media. II. Transition to turbulent regime, Chem. Eng. Sci. 53(22) (1998) 3897-3909.

[27] S. Wardeh, H.P. Morvan, CFD simulations of flow and concentration polarization in spacer-filled channels for application to water desalination, Chem. Eng. Res. Des. 86 (2008) 1107-1116. 